\title{
Optimization design for aerodynamic elements of high speed trains
}

\author{
Yao Shuanbao a, Guo Dilong ${ }^{a}$, Sun Zhenxu ${ }^{\mathrm{a}, *}$, Yang Guowei ${ }^{\mathrm{a}}$, Chen Dawei ${ }^{\mathrm{b}}$ \\ ${ }^{a}$ Key Laboratory for Mechanics in Fluid Solid Coupling Systems Institute of Mechanics, Chinese Academy of Sciences, Beijing 100190, China \\ ${ }^{\mathrm{b}}$ CSR Qingdao Sifang Locomotive Co., Ltd., Qingdao 266111, China
}

\section{A R T I C L E I N F O}

\section{Article history:}

Received 11 April 2013

Received in revised form 25 October 2013

Accepted 22 February 2014

Available online 3 March 2014

\section{Keywords:}

Wake flow

Multi-objective optimization

Cross-validation

Kriging

High speed trains

\begin{abstract}
A B S T R A C T
The complex wake flow of high speed trains severely influences the running safety and amenity of the trailing car. In this paper, based on the streamlined shape of CRH380A high speed train, taking the aerodynamic lift force of the trailing car and the volume of the streamlined head as the objectives, an efficient multi-objective optimization process based on the response surface has been constructed. The Kriging model has been constructed based on the cross-validation method and genetic algorithm (GA). This approach could decrease the number of training samples and improve the optimization efficiency while without decreasing its generalization. After the Pareto optimal solutions being obtained, four design points are chosen for comparative study with the original shape, and one of these points is chosen for the unsteady aerodynamic study together with the original shape. The results reveal that the variation trends of the lift force and the side force of the trailing car are the same as that of the drag of the whole train. After optimization, the volume of the streamlined head is almost the same as that of the original shape. Compared to the original shape, the lift force of the trailing car decreases by $27.86 \%$ of and the drag of the whole train decreases by $3.34 \%$ in conditions without crosswind, and the lift force of the trailing car decreases by $5.43 \%$, the side force of the whole train decreases by $72.09 \%$ and the drag of the whole train decreases by $2.1 \%$ in the crosswind conditions. The optimal train benefits from low fluctuations of lift and side force of the trailing car. Besides, better wake flow could be obtained, and the wake vortices are suppressed, too. Consequently, the running safety and amenity of HST are improved a lot after optimization.
\end{abstract}

(c) 2014 Elsevier Ltd. All rights reserved.

\section{Introduction}

As a large length-to-diameter ratio transportation vehicle, high speed trains (HST) run quicker and quicker. The speed of HST that running on the Beijing-Shanghai railway has already reached $300 \mathrm{~km} / \mathrm{h}$. In high speed conditions, the flow around the train reveals distinct unsteady characteristics due to the influence of the exposed structures (bogies, pantographs, etc.) and the ground effect [1]. Furthermore, the unsteady characteristics of the wake flow for a slender body are more predominant, which may lead to high fluctuations of aerodynamic loads, especially for the lift and side force of the trailing car. Large aerodynamic lift force will decrease the contact force between the wheels and the rail, which will lead to derailment in certain circumstance. For the flow around a slender body, asymmetric wake flow will emerge in crosswind conditions, and the shedding vortices around the nose of the trailing car will result in high lift and side force. Meanwhile, enough space should be ensured not only for the placement of equipments but also for the operation convenience of the drivers.

\footnotetext{
* Corresponding author. Tel.: +86 01082543815.

E-mail address: sunzhenxu@imech.ac.cn (S. Zhenxu).
}

Consequently, it is very necessary to ensure enough space of the streamlined head during the shape optimization of HST.

The streamlined part is very essential to the aerodynamic performance of HST. The aerodynamic performance could be effectively improved by optimizing the train shape [1]. However, the running environment of HST is very complex, and lots of design objectives (such as the aerodynamic drag force, the aerodynamic lift force of the trailing car, aerodynamic side force of the trailing car, the aerodynamic overturning moment of the trailing car, the aerodynamic rolling moment of the trailing car, the micro-pressure wave generated when the train pass a tunnel, the pressure wave generated when two trains crossing each other on a double railway and the aerodynamic noise.) have a serious effect on the running safety and amenity of HST. Dozens of design parameters are needed to accurately control the geometry of the high-speed train head, and several geometric constraints are also required to ensure the usefulness of the train head. Meanwhile, the aerodynamic shape optimization needs large amount of computational cost and hundreds of thousands times of flow field calculations should be required for one optimization. Thus, it is unbearable to take all of the design objectives into consideration when optimizing a train head. Limited by the computer technique and optimization 
algorithms, early studies on the streamline shape optimization of HST mainly rely on wind tunnel experiments and numerical simulations [2]. Meanwhile some methodology studies on two-dimensional profile of the streamlined head have been performed with gradient algorithms [3]. In order to reduce the computational cost and shorten the optimization cycle, some scholars introduce the response surface method (RSM) into the aerodynamic optimization [2-10]. In recent years, great progress has been obtained both for the response surface technique and the computer technique, which greatly improves the optimization efficiency and makes the engineering optimization of HST be possible.

Jongsoo and Junghui [3] conducted a two-dimensional optimization on reducing the micro-pressure wave with the RSM technique and sequential quadratic programming algorithm. Vytla et al. [4] performed a two-dimensional optimization study on minimizing the aerodynamic drag and noise based on the Kriging model and GA-PSO hybrid algorithm. In order to obtain the micro-pressure wave when the train passes by tunnel, both Jongsoo and Vytla $[3,4]$ used axial symmetric equations to simulate the flow field. Combined with GA and arbitrary shape deformation technique, Sun et al. [11] performed a three-dimensional aerodynamic shape optimization of HST. In order to reduce the computational cost, only the streamlined head has been considered in Ref. [11], which may decrease the computational accuracy of the flow field. Ku et al. [5] utilized the polynomial RSM to optimize the micro-pressure wave and obtained the optimal cross-sectional area distribution. Taking this distribution as the constraint, they then performed the three-dimensional optimization on minimizing the aerodynamic drag based on Kriging model. However, the whole process still belongs to a single objective optimization. Krajnovic [6] took the drag force coefficient, rolling moment coefficient and yawing moment coefficient as objectives to optimize a very simple train front for crosswind stability. Three kinds of RSM are adopted to reduce the CFD cost in his work. Because of difference of the three RSM methods prediction accuracy, Krajnovic found that the performance of the combination of RBNN and polynomial functions is better than the two others (polynomial functions and radial basis neural networks). Besides, in Ref. [6], Krajnovic also optimized the vortex generators to reduce the drag of the train. The study inspires us to make full use of RSM to reduce the CFD cost. In conclude, the aerodynamic shape optimization of HST found in the above references mainly focus on two-dimensional profiles or very simple three-dimensional shapes, and they mainly belong to methodology study and could hardly be used to engineering problems.

Thus, the main propose of our work in this paper is to introduce the popular optimization algorithms and RSM technique into engineering problems with amounts of CFD cost, adopt a procedure to shorten the design cycle of new streamlined parts of HST as much as possible, and shed light on aerodynamic shape design based on the unsteady flow field. Aerodynamics design objectives that seriously influence the running safety and amenity of HST (such as the aerodynamic lift force of the trailing car, aerodynamic side force of the trailing car, the aerodynamic overturning moment of the trailing car, the aerodynamic rolling moment of the trailing car, the pressure waves generated when the train pass a tunnel) are considerate in this paper. Besides, the aerodynamic drag force is also considerate in our work to make sure that the optimal train is friendly to environment. However, some other design objectives (such as two trains crossing each other on a double railway and the aerodynamic noise) are not discussed in this paper. Only the lift force of the trailing car and the volume of streamlined part are taken to be optimal objectives so as to reduce the CFD cost and make the optimal problem be practical. So we mainly take the shape of the trailing car into consideration in this paper and discuss the influence of the leading car shape to pressure waves in Section 6.4 carefully. The Kriging model is adopted together with the multi-points criterion based on minimum response surface method to reduce the CFD cost and improve the optimization efficiency. Meanwhile, a cross-validation training approach has been proposed in this paper which greatly reduces the number of training samples. Based on the Kriging model of which the accuracy meets the engineering requirement, the Pareto front has been obtained with NSGA-II. Then four typical design points are chosen from the Pareto solutions for comparative study with the original shape known as CRH380A, and one of these points is chosen for the unsteady aerodynamic study together with the original shape.

\section{Optimization process}

Although the RSM has been utilized, the design cycle of the streamlined parts of HST is still very long. Any unreasonable operating during the optimization process may deteriorate the optimization solutions, even result in disagreeable solutions. Thus, the optimization process should be designed reasonably and reduce unnecessary steps as many as possible. The whole optimization process in the present paper is listed as below, as Fig. 1 shows:

(1) Determine the design variables and their ranges based on the optimization problems.

(2) Determine the number of training samples which could meet the requirement of the RSM model, sampling in the design space with the use of central Latin hypercube sampling method with maximin criteria, obtain the initial value of the design variables for each training sample.

(3) Get the exact value of objectives for each training sample with CFD tools; the values of some objectives could be obtained by other approaches, the volume of the streamlined part, for instance.

(4) Train the Kriging model based on the cross-validation method and the real-coded GA.

(5) Based on the Kriging model, optimization is performed with the multi-objective non-dominated sorting genetic algorithm, and the Pareto front is obtained.

(6) Choose some samples from the Pareto solutions as the testing samples, and CFD validation is performed to judge whether the predicting accuracy has been achieved.

(7) If the predicting accuracy of the testing samples is not met, these samples should be added to the training sample set, return to step (3), and reconstruct the Kriging model.

(8) If the predicting accuracy is achieved, the Kriging model is recognized as correctly constructed. The Pareto solutions are then the final optimal solutions.

\section{Parametric methods}

\subsection{Geometry}

Optimization of the head of HST mainly focuses on the streamlined part, of which the key design variables are as follows: the cross-sectional area distribution and the slenderness ratio of the streamlined part, the longitudinal-type line and horizontal-type line of the streamlined part, the drainage around the nose, the cab perspective and the bogie shield. In the present paper, a CRH380A 1:1 model with three carriages has been utilized for aerodynamic shape optimization. The connection part between adjacent carriages is completely eliminated. Meanwhile, the cavities around the bogies are all closed for simplification. Considering that the bogie below the trailing streamlined part is crucial for the wake flow and the flow below the trailing car, this bogie is 


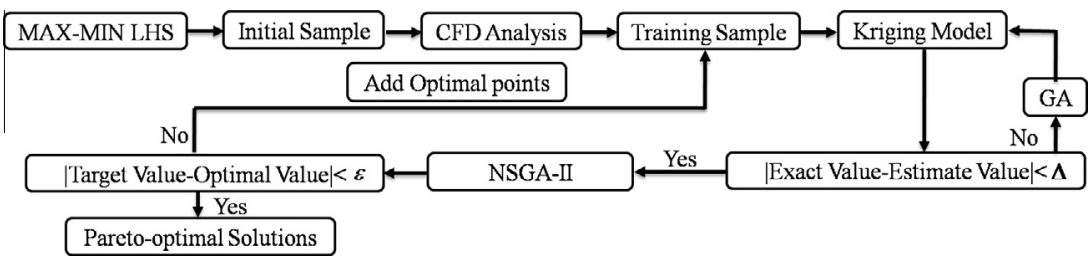

Fig. 1. The optimization process.

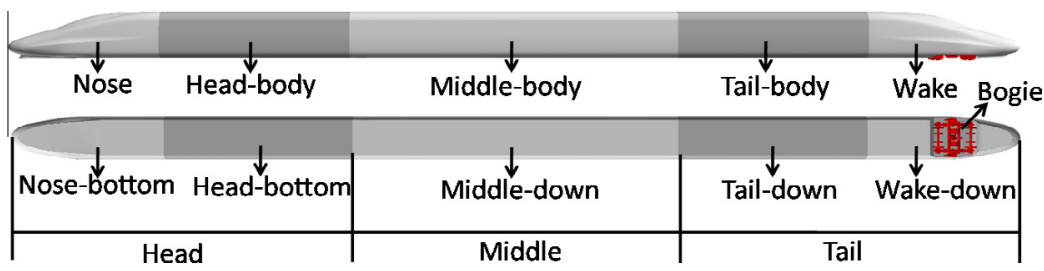

Fig. 2. The geometric parameters of the simplified shape of CRH380A.

preserved to precisely predict the lift force of the trailing car, as Fig. 2 shows.

The geometric parameters of the simplified shape of CRH380A are as follows: the length of the leading car and trailing car is $26.5 \mathrm{~m}$, and the shape of the trailing car is the same as the leading car. The length of the middle car is $25 \mathrm{~m}$. The length of the streamlined part is $12 \mathrm{~m}$, the height is $3.5 \mathrm{~m}$, the width is $3.38 \mathrm{~m}$, the maximum cross-sectional area is $11.2 \mathrm{~m}^{2}$ and the slenderness ratio is 3.55. The type of the cross-sectional area distribution of CRH380A is a three-sectional type, of which the longitudinal-type line is double-arch, the horizontal-type line is flat spindle and the nose is of ellipsoid.

\subsection{Parametric approach}

In order to correctly describe the deformation of the streamlined part of HST, a parametric approach based on the idea of arbitrary shape deformation has been adopted in the present paper. The main idea of the approach can be found in Ref. [7]. As Fig. 3 shows, five deformation zones have been extracted from the CRH380A streamlined part. For each deformation zone, mesh discretization is firstly applied and specific node coordinates are obtained. Then the surface deformation could be achieved with the use of deformation functions. The deformation functions of each deformation zone are listed as follows:

$$
\text { ZONE1: } \begin{aligned}
\Delta y= & w_{1} \cos \left(\pi\left(x-x_{1}\right) /\left(m x_{1}-n x_{1}\right)\right) \\
& \times \cos \left(\pi\left(y_{1}-\text { menum }\right) /\left(m y_{1}-n y_{1}\right)\right)
\end{aligned}
$$

where $\Delta y$ is the increment of $y$ coordinate of grids in ZONE1, and $w_{1}$ is the first design parameter, which controls the deformation of the nose drainage zone. $x_{1}$ and $y_{1}$ are the $x$ and $y$ coordinate of control point 1 , respectively. $x$ is the $x$ coordinate of grids in ZONE1. menum is the grid number coordinate of $z$ coordinate axis, which is an integer parameter. $m x_{1}$ and $n x_{1}$ are the maximal and minimal value of $x$ coordinate of grids in ZONE1. $m y_{1}$ and $n y_{1}$ are the maximal and minimal value of $y$ coordinate of grids in ZONE1.

$$
\text { ZONE2: } \Delta z=w_{2} \cos \left(\pi\left(x-x_{2}\right) /\left(m x_{2}-n x_{2}\right)\right)
$$$$
\times \cos \left(\pi\left(z-z_{2}\right) /\left(m z_{2}-n z_{2}\right)\right)
$$

where $\Delta z$ is the increment of $z$ coordinate of grids in ZONE2, and $w_{2}$ is the second design parameter, which controls the height of the nose drainage zone. $x$ and $z$ are the $x$ and $z$ coordinate of grids in ZONE2. $x_{2}$ and $z_{2}$ are the $x$ and $z$ coordinate of control point 2 . $m x_{2}$ and $n x_{2}$ are the maximal and minimal value of $x$ coordinate of grids in ZONE2. $m z_{2}$ and $n z_{2}$ are the maximal and minimal value of $z$ coordinate of grids in ZONE2.

$$
\begin{array}{cc}
\Delta y=w_{3} \cos \left(\pi\left(x-x_{3}\right) /\left(m x_{3}-n x_{3}\right)\right) \\
\text { ZONE3 : } \quad \times \sin \left(\pi\left(y-y_{3}\right) /\left(2\left(m y_{3}-n y_{3}\right)\right)\right) * \\
\cos \left(\pi\left(z-z_{3}\right) /\left(2\left(m z_{3}-n z_{3}\right)\right)\right)
\end{array}
$$

where $\Delta y$ is the increment of $y$ coordinate of grids in ZONE3, and $w_{3}$ is the third design parameter, which controls the width of the train body. $x, y$ and $z$ are the $x, y$ and $z$ coordinate of grids in ZONE3. $x_{3}, y_{3}$ and $z_{3}$ are the coordinates of control point 3. $m x_{3}$ and $n x_{3}$ are the maximal and minimal value of $x$ coordinate of grids in ZONE3. $m y_{3}$ and $n y_{3}$ are the maximal and minimal value of $y$ coordinate of grids in ZONE3. $m z_{3}$ and $n z_{3}$ are the maximal and minimal value of $z$ coordinate of grids in ZONE3.

$$
\text { ZONE4 }: \begin{aligned}
\Delta z= & w_{4} \cos \left(\pi\left(x-x_{4}\right) /\left(m x_{4}-n x_{4}\right)\right) \\
& \times \sin \left(\pi\left(z-z_{4}\right) /\left(2\left(m z_{4}-n z_{4}\right)\right)\right)
\end{aligned}
$$

where $\Delta z$ is the increment of $z$ coordinate of grids in ZONE4, and $w_{4}$ is the fourth design parameter, which controls the cab perspective. $x$ and $z$ are the $x$ and $z$ coordinate of grids in ZONE4. $x_{4}$ and $z_{4}$ are the
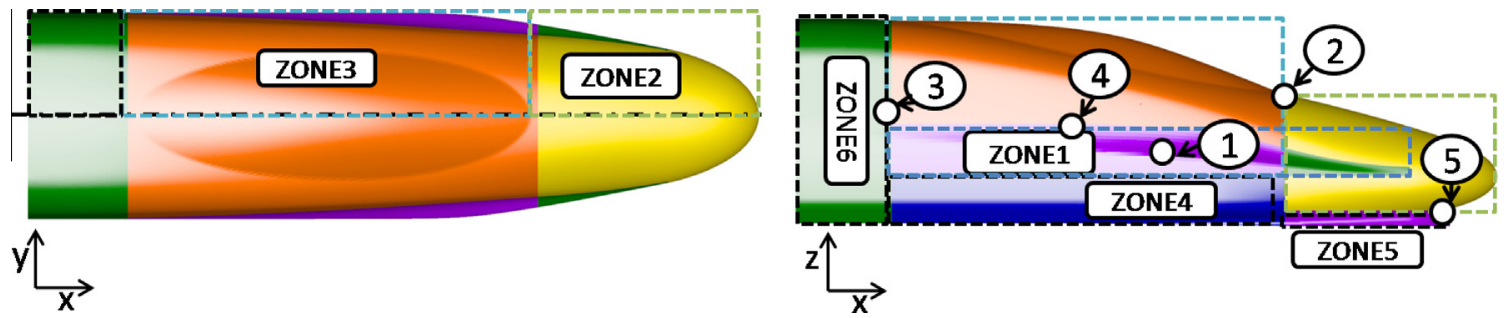

Fig. 3. Schematic diagrams of deformation zones. 
Table 1

The ranges of the design parameters.

\begin{tabular}{llllll}
\hline $\begin{array}{l}\text { Design } \\
\text { parameters }\end{array}$ & $\begin{array}{l}\mathrm{W} 1 \\
(\mathrm{~mm})\end{array}$ & $\begin{array}{l}\mathrm{W} 2 \\
(\mathrm{~mm})\end{array}$ & $\begin{array}{l}\mathrm{W} 3 \\
(\mathrm{~mm})\end{array}$ & $\begin{array}{l}\text { W4 } \\
(\mathrm{mm})\end{array}$ & $\begin{array}{l}\text { W5 } \\
(\mathrm{mm})\end{array}$ \\
\hline Ranges & $(-8,8)$ & $(-20,15)$ & $(-10,25)$ & $(-15,5)$ & $(-100,15)$ \\
\hline
\end{tabular}

$x$ and $z$ coordinate of control point $4 . m x_{4}$ and $n x_{4}$ are the maximal and minimal value of $x$ coordinate of grids in ZONE4. $m z_{4}$ and $n z_{4}$ are the maximal and minimal value of $z$ coordinate of grids in ZONE4.

$$
\text { ZONE5 : } \begin{aligned}
\Delta x= & w_{5} \cos \left(\pi\left(x-x_{5}\right) /\left(2\left(m x_{5}-n x_{5}\right)\right)\right) \\
& \times \sin \left(\pi\left(z-z_{5}\right) /\left(m z_{5}-n z_{5}\right)\right)
\end{aligned}
$$

where $\Delta x$ is the increment of $x$ coordinate of grids in ZONE5, and $w_{5}$ is the fifth design parameter, which controls the shape of the cowcatcher. $x$ and $z$ are the $x$ and $z$ coordinate of grids in ZONE5. $x_{5}$ and $z_{5}$ are the $x$ and $z$ coordinate of control point 5. $m x_{5}$ and $n x_{5}$ are the maximal and minimal value of $x$ coordinate of grids in ZONE5. $m z_{5}$ and $n z_{5}$ are the maximal and minimal value of $z$ coordinate of grids in ZONE5.

The zones are deformed one by one. After obtaining all the coordinate increments of the deformation zones, the new designed surface then could be obtained. For convenience of parameterization, the length of the streamline is normalized as $1 \mathrm{~m}$. In order to keep enough space for the placement of ancillary parts, the width of the bottom of the train and the length of the streamlined part are kept unchanged when parameterization is performed. The ranges of the five design parameters are listed as Table 1 shows.

\section{Numerical method}

\subsection{Rans}

CFD accuracy that directly affects the construction of the Kriging model and efficiency of optimization algorithm is the foundation for the whole optimization process. In this paper, the speed of high-speed train is $300 \mathrm{~km} / \mathrm{h}$, so the Mach number is 0.245 . Under this condition, the air compression characteristic has an obvious effect on the aerodynamic performance of HST. Therefore, the steady compressible Reynolds-averaged Navier-Stokes equations [12] based on the finite volume method are used to predict the aerodynamic force and moments. Roe's FDS scheme is used to calculate convective flux and Lower-Upper Symmetric GaussSeidel (LU-SGS) is chosen for temporal discretization. The $k-\omega$ SST model is selected as the turbulence model. The standard wall functions [13] are used near the wall so that the accuracy of the CFD results could be ensured with a limited amount of mesh.

Computational domains and boundary conditions: taking the length of the simplified train as the characteristic length $L$, then the length of inflow direction is $1 L$, the length of outflow direction is $2 L$, the width is $1 L$, the distance between the bottom of the train and the ground is $0.00235 \mathrm{~L}$ and the height is $0.5 \mathrm{~L}$, as shown in Fig. 4. The flow velocity is $83.33 \mathrm{~m} / \mathrm{s}$; the far-field pressure is $1 \mathrm{~atm}$, the temperature is $288 \mathrm{~K}$ and the reference area is the maximum cross-sectional area of the train. Thanks to the compressibility calculation model, the one-dimensional inviscid flow of the Riemann invariants is introduced as the far-field boundary conditions, which are also known as non-reflective boundary conditions. Inflow, outflow and the top boundaries are all set as far-field boundary conditions and the train body is a non-slip wall. The ground is treated as the moving wall so as to simulate the ground effect, and the moving speed is equal to the train speed.

\section{2. $D E S$}

Compared with the steady flow calculation, the unsteady approach needs much more computational time. For a single training sample, five days are required for the unsteady aerodynamic calculation even in the case that $60 \mathrm{CPUs}$ are utilized. In order to correctly construct the Kriging model, at least 20 samples are needed, indicating that 30 times more or less of flow field calculations are required. Consequently, the aerodynamic shape optimization of high speed trains cannot directly utilize the unsteady results.

Although huge computational cost is required for the unsteady calculation, it is very necessary to perform unsteady analysis for the optimization results. It can be easily learned the flow field details via unsteady analysis, and the flow mechanism and flow structures around the train can also be obtained. Meanwhile, the unsteady time history for aerodynamic loads could also be obtained. It is the objective to improve the flow performance in the wake zone and then improve the running safety and amenity of HST.

The detached eddy simulation (DES) is a hybrid method which combines RANS and LES together [14,15], and benefits a lot in solving large separation problems with high Reynolds numbers. DES usually keeps high accuracy in predicting aerodynamic drag and separation locations for large separation flows around ground transport vehicles. With the help of DES, the complicated trailing vortices in the wake zone could be precisely captured. The most popular DES approaches could be divided into two kinds: one is based on the SA model and the other one is based on the $k-w$ SST turbulence model, which is used in the present paper. For the latter one, it is realized by replacing the turbulence length scale factor $n$ with the function, in which: $\Delta_{\max }=\max [\Delta x, \Delta y, \Delta z]$, where $\Delta x, \Delta y, \Delta z$ are the lengths of the sides of the controlling volume respectively. As a result, the transition between URANS and LES could be achieved [14].

The finite volume method based on cell center is adopted for the discritization of the controlling equations. A second order upwind scheme is used for convection terms. While for viscous terms, the second order central differentiation scheme is used. A fully implicit scheme is adopted for time discritization. The time step used in the simulation is $0.0005 \mathrm{~s}$ and the inner steps are
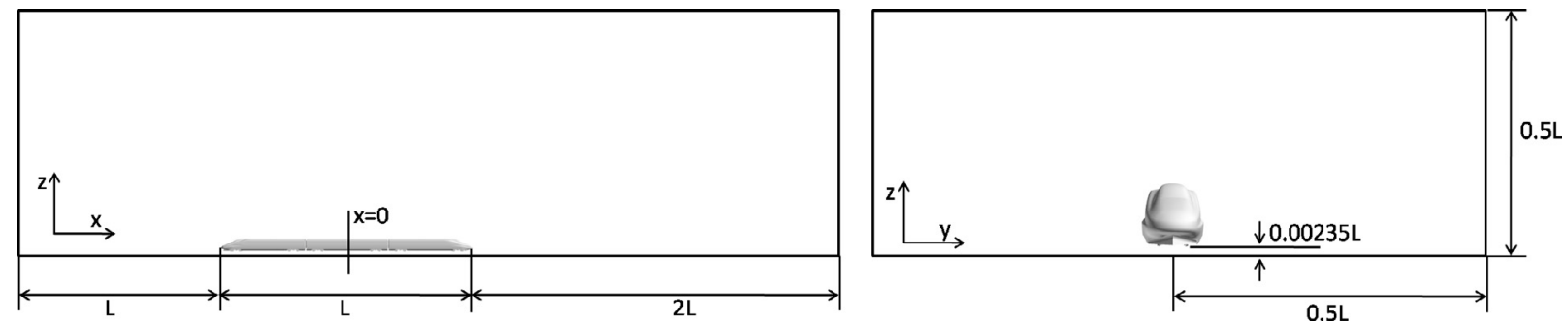

Fig. 4. Computational domain 
chosen as 5. Meanwhile, the standard wall function is used for near wall treatment. In order to facilitate the comparison with the steady results, both the steady analysis and the unsteady analysis are utilizing the same mesh, of which 6 prisms are utilized around the train. The value of $y+$ around the train ranges from 30 to 50 , and the minimum value of the grid in the computational domain is $120 \mathrm{~mm}$. The whole amount of the grids is about 11 million.

\subsection{CFD validation}

In order to facilitate the comparison of aerodynamic loads, all the aerodynamic loads are expressed by force coefficients, which are listed as follows:

The pressure coefficient $C_{p}$,

$C_{p}=\frac{2 P}{\rho V^{2}}$

The drag coefficient $C_{d}$,

$C_{d}=\frac{2 F_{x}}{\rho V^{2} S_{x}}$

The lift coefficient $C_{l}$,

$C_{l}=\frac{2 F_{z}}{\rho V^{2} S_{x}}$

The side force coefficient $C_{S}$,

$C_{s}=\frac{2 F_{y}}{\rho V^{2} S_{x}}$

The coefficient of overturning moment $m_{x}$,

$m_{x}=\frac{M_{x}}{\frac{1}{2} \rho V^{2} S_{x} L}$

The coefficient of pitching moment $m_{y}$,

$m_{y}=\frac{M_{y}}{\frac{1}{2} \rho V^{2} S_{x} L}$

The coefficient of rolling moment $m_{z}$,

$$
m_{z}=\frac{M_{z}}{\frac{1}{2} \rho V^{2} S_{x} L}
$$
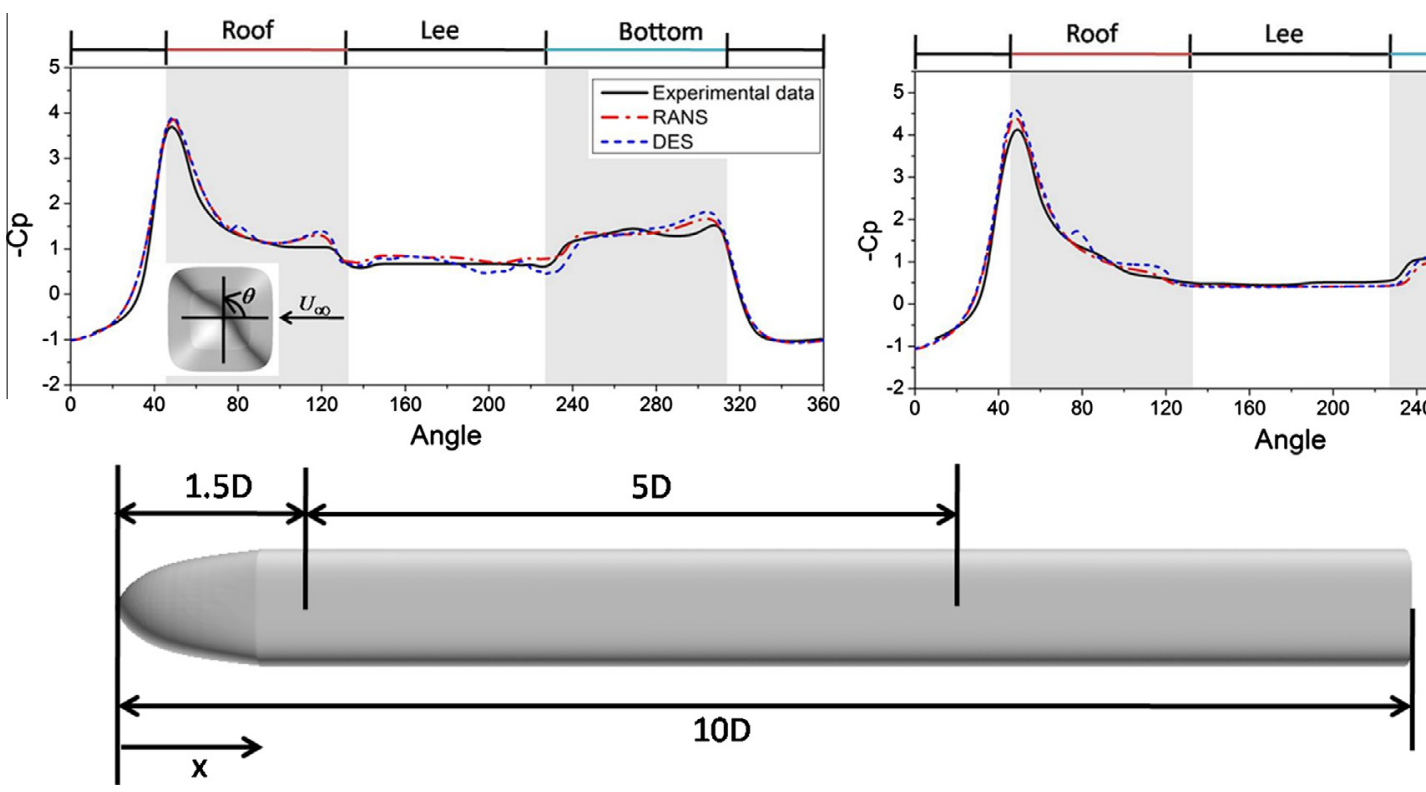

In which, $P$ is the dynamic pressure, $F_{x}$ is the aerodynamic drag, $F_{z}$ is the aerodynamic lift, $F_{y}$ is the side force, $\rho$ is the density of the air, $V$ is the inflow velocity, which is the running speed of the train in conditions without crosswind, and also is the resultant velocity of the running speed of the train and the velocity of the crosswind in crosswind conditions. $S_{x}$ is the reference area, which is the maximum cross-sectional area of the train $\left(11.2 \mathrm{~m}^{2}\right) . L$ is the reference length, which is $3.5 \mathrm{~m}$. The moment center for the overturning moment is the wheel-rail contact point, and the moment center for the pitching moment and rolling moment is the centroid of the train.

The wind tunnel experiment (for the long nose) conducted by Chiu [16] has been utilized in the present paper for the validation of numerical algorithms. Hemida and Krajnovic [17] performed a systematic study on this model by the large eddy simulation approach (LES) and abundant comparative study with the experimental data has been performed. In the present paper, both the RANS and DES approach have been adopted to simulate the flow around the wind tunnel model. The Reynolds number is around 300,000 based on the inflow velocity and the height of the model. The computational domain is the same with the domain that Hemida uses. The Cartesian grids are generated in the domain with an amount of 4.8 million. Fig 5 shows the pressure coefficient distribution in the section predicted by the two approaches. It can be seen that better vortex structures could be simulated by DES, leading that the fluctuation of the pressure coefficient is stronger that predicting by RANS. The numerical results agree well with the experimental results. Although RANS model predicts worse than the DES model in fine structures in the flow field, its accuracy still meets the requirement in engineering. In order to improve the optimization efficiency, the RANS model has been adopted for the CFD analysis for each training sample, while the DES model is adopted for the unsteady aerodynamic analysis for the optimal solutions.

\subsection{Analysis for different simplified CRH380A models}

In order to validate the reasonability of Model-I, another two models, Model-II (a simplified model, eliminating all the bogies and connection parts between adjacent carriages) and Model-III (a real model without any simplifications), have been considered in the present paper. Table 2 shows the results of these models. It can be seen that big difference of aerodynamic drag exists among
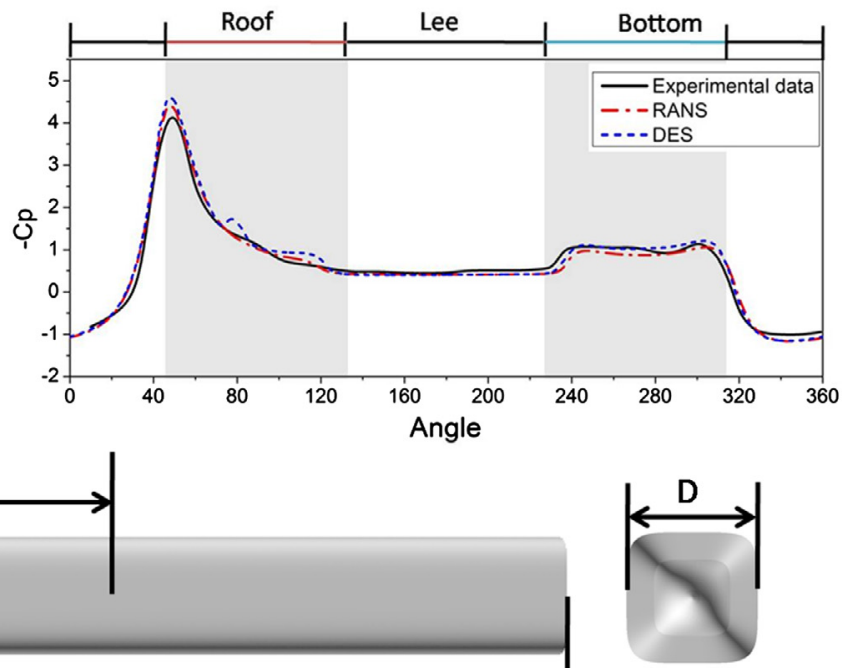

Fig. 5. Local surface pressure coefficient, at $x / D=1.5$ and $x / D=6.5$ (the model is copied from Ref. [17] and the style of the figure is the same as shown in Ref. [17]). 
Table 2

Computational results of the three simplified models.

\begin{tabular}{llrll}
\hline Model type & Total $C_{d}$ & \multicolumn{1}{c}{ Tail $C_{l}$} & Pressure drag & Shear drag \\
\hline Model-I & 0.1971 & 0.0546 & 0.0660 & 0.1311 \\
Model-II & 0.1567 & -0.0237 & 0.0231 & 0.1336 \\
Model-III & 0.3224 & 0.0366 & 0.2146 & 0.1078 \\
\hline
\end{tabular}

the models, but they are all positive. However, the lifts of Model-I and Model-III on the trailing car keep positive while that of Model-II keeps negative. It can be concluded that the last bogie below the trailing car influences significantly on the aerodynamic performance of the trailing car. Compared to Model-II, Model-I could better reveal the aerodynamic characteristics of the real train, indicating that Model-I is a more reasonable simplified model.

\section{Kriging model based on cross-validation}

\subsection{Construction of Kriging model}

The Kriging surrogate model is an interpolation model based on statistical theory [18]. Compared to the polynomial RSM model, the Kriging model could obtain more precise global optimal results. This model is composed of regression model and related model. The former is a global approximation in the design space, while the latter could reflect the variables' distribution structure in the design space, which impacts directly on the predicting accuracy of the model. The essence of constructing the Kriging model is to find the optimal value of $\theta_{i}$, and turn the solution of into the nonlinear unconstrained maximum optimization problem. However, this approach has not been adopted in the present paper, but the crossvalidation approach has been considered instead. $\theta_{i}$ is calculated directly by minimizing the predicting error of training samples. For a multi-objective optimization problem, the calculation of $\theta_{i}$ is a multi-objective, multi-variable process. In order to simplify this problem, taking the objective with the maximal variation as the main objective, a single-objective optimization for $\theta_{i}$ is utilized in the present paper. The optimal value could be obtained with the realcoded GA approach. In order to fully utilize the information of sample points and reduce the number of training samples, an approach based on the combination of cross-validation method and the GA method has been proposed in the present paper to construct the Kriging model, of which the process is listed as follows:

(1) Determine the main objective according to the variation of all the objectives.

(2) Determine the range of $\theta_{i}$ according to the influence to the objectives by the design variables.

(3) Initialize the parameters required by GA, such as the population size, selection probability, crossover probability, mutation probability, the initial population and the biggest evolutionary generation.

(4) Divide the training points into $N$ groups randomly, and the number of training points in each group should not be too much, so as to make full use of each training point.

(5) Take the training points in $N-1$ group to construct a subKriging model (relevant parameter of each individual is $\theta_{i}$ ), and take the last one group as the validation sampling points. Keep $\theta_{i}$ unchanged, take each group as the validation group sequentially.

(6) Take the average value of the predicting error error $_{i}$ as the objective of GA.

(7) The best value of $\theta_{i}$ is found with GA through minimum of $\sum_{i=1}^{N} \mid$ error $_{i} \mid / N$, and then take the sub-Kriging model with the minimal predicting error as the final model.

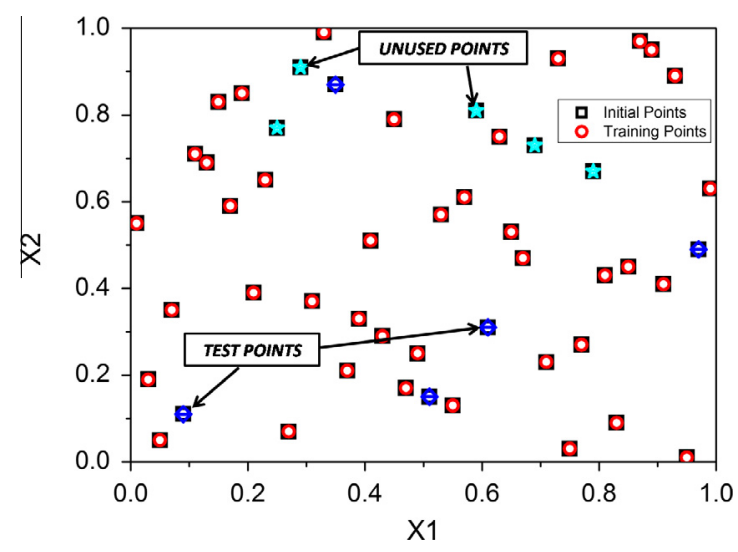

Fig. 6. The distribution of sample points in the design space.

It could be seen that the computational time to construct the Kriging model based on the cross-validation algorithm is rather longer than that from conventional approaches. However, compared to the computational cost of flow field calculations of each training point, this could be neglected. Consequently, the approach proposed in this paper would be favorable for aerodynamic shape optimization with large computational cost.

\subsection{Validation}

In order to verify the efficiency of the approach proposed in this paper, the following test function [19] (minimization problem) is chosen for analysis:

TEST: $\left\{\begin{array}{l}f_{1}(\boldsymbol{x})=x_{1} \\ f_{2}(\boldsymbol{x})=\left(1+10 x_{2}\right)\left[1-\left(\frac{x_{1}}{1+10 x_{2}}\right)^{2}-\frac{x_{1}}{1+10 x_{2}} \sin \left(8 \pi x_{1}\right)\right] ; \quad 0 \leqslant x_{1}, \quad x_{2} \leqslant 1 。\end{array}\right.$

The Pareto front of the above test function is a non-continuous solution set, and several extreme points exist, which is commonly used to validate the searching capability for multi-objective optimization algorithms. The first objective is linear to the first variable while strong non-linear characteristics exist between the second objective and the second variable, which could be utilized to validate the fitting accuracy of the Kriging model.

Central Latin hypercube sampling method with maximin criteria based on the iterative local search algorithm [20] is adopted and 50 sample points are chosen in this paper. Taking 45 points as the training points and the rest 5 points as validation points. Fig. 6 shows the distribution of training points and validation points in the design space. It could be seen that they are all evenly distributed.

For the construction of cross-validation Kriging model (CV-Kriging model), $\theta_{i}$ ranges from 0 to 10 , the initial population size is 100 , the evolution generation is set to 1000 , the crossover probability is 0.9 and the mutation probability is 0.3 . The training points are evenly divided into 5 groups. Fig. 6 shows the final 5 unused points (UNUSED POINTS). These points locate in the zone where $\mathrm{X} 2$ is relatively larger. The typical Kriging model is constructed by pattern searching approach (PS-Kriging model), which is very sensitive to the initial value of $\theta_{i}$. Fig. 7 shows the Pareto optimal results from theoretical solution, PS-Kriging model and CV-Kriging model. It could be seen that results from CV-Kriging model agree well with the theoretical solution, while bad agreement from the PS-Kriging model. The results reveal that CV-Kriging model obtains stronger capability for the test function. More efficient construction approach will utilize relatively few sample points, which will aid in aerodynamic shape design with huge computational cost. 


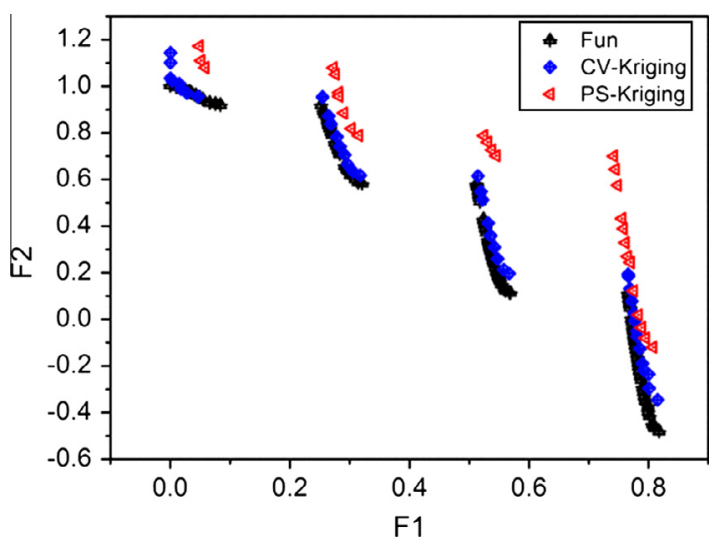

Fig. 7. Pareto solutions of the test function gotten by different methods.

The CV-Kriging model could fully utilize the information about training points and reduce the number of training points while keeping the predicting accuracy unchanged.

\subsection{Construction of Kriging model used for high speed train nose optimization}

Considering the huge computational cost in the aerodynamic optimization, especially in the shape optimization of HST, a sequential optimization method based on minimizing the response surface criteria [18] has been adopted in this paper so as to reduce the training points. A basic Kriging model is firstly constructed from the initial 20 training points. Based on this model, the Pareto set is obtained. Then four typical points of the Pareto set are chosen for CFD validation. If the optimization accuracy does not meet the requirement, these points then should be added to the initial training set so as to build the Kriging model much more accurately. If the optimization accuracy meets the design requirement, the Kriging model is finally constructed. During each iteration process, the training points are divided into groups randomly, and each group has 2 training points. The Kriging model is obtained based on the cross-validation algorithm. The volume of the streamlined part hardly varies in the design space while the lift force of the trailing car varies apparently. With the use of the same training points, the prediction accuracy of the Kriging model for the volume could limit its error within $1 \%$ while the accuracy for the lift force of the trailing car only limits its error within $5 \%$.

The lift force of the trailing car $C_{l}$ and the volume of the streamlined part Vol are chosen as the objectives in this paper,

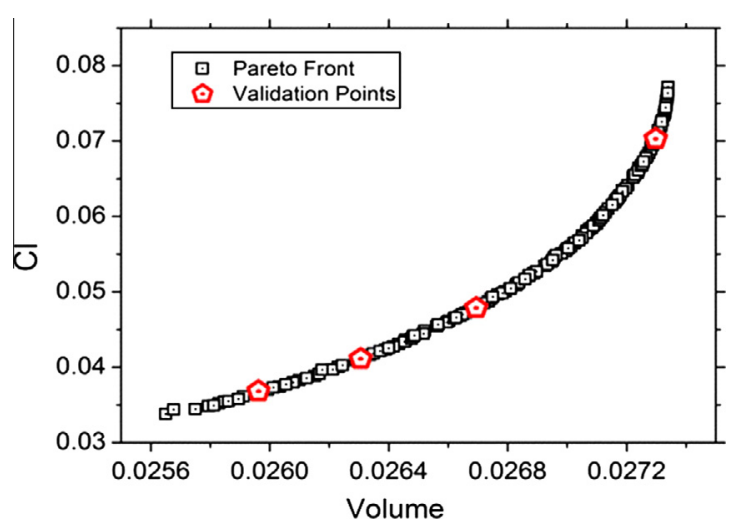

Fig. 9. The Pareto solution that is found based on initial sample points.

Table 3

The predication values and CFD values of test points.

\begin{tabular}{lllllll}
\hline & Real Vol & Kriging & Error $(\%)$ & Real $C_{l}$ & Kriging & Error $(\%)$ \\
\hline TEST1 & 0.02597 & 0.02596 & 0.004 & 0.03968 & 0.03684 & 7.157 \\
TEST2 & 0.02630 & 0.02631 & 0.004 & 0.04340 & 0.04112 & 5.253 \\
TEST3 & 0.02670 & 0.02669 & 0.004 & 0.04881 & 0.04789 & 1.885 \\
TEST4 & 0.02756 & 0.02730 & 0.943 & 0.07258 & 0.07031 & 3.128 \\
\hline
\end{tabular}

and $C_{l}$ varies larger than Vol. Thus, $C_{l}$ is chosen as the main objective during the training of the Kriging model. As parameters for GA operation, the size of initial population is set to 100 . The evolution generation is set to 5000 . The crossover probability is 0.9 and the mutation probability is 0.3 , respectively. All the values of $\theta_{i}$ range from 0 to 10 . Fig. 8 shows the convergence history of the fitness of the optimal solutions after adding training points. It could be seen that the fitness converges slowly during the iterations and fluctuates greatly, but finally it could converge to a constant value, indicating that the cross-validation algorithm increase the complexity of the problem, and yet still could construct a stable Kriging model.

Fig. 9 shows the Pareto optimal set obtained by the CV-Kriging model, and four points from this set are chosen for CFD validation. Table 3 shows the results from the CV-Kriging model and CFD simulations. It could be seen that the predicting error for Vol from the Kriging model has already met the engineering requirement while the predicting error for $C_{l}$ still not meets the requirement. Consequently, these two sampling points are taken into the set of training points and the Kriging model is trained again. After adding the points, the predicting errors for the two objectives both meet the requirement, which will be discussed in the following section.

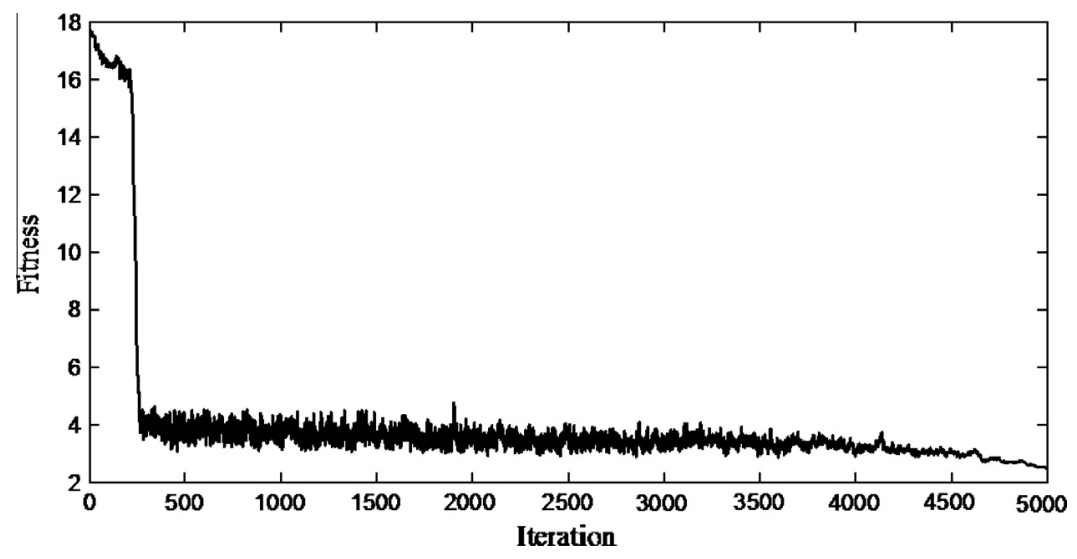

Fig. 8. History of average fitness value along with the evolution number. 


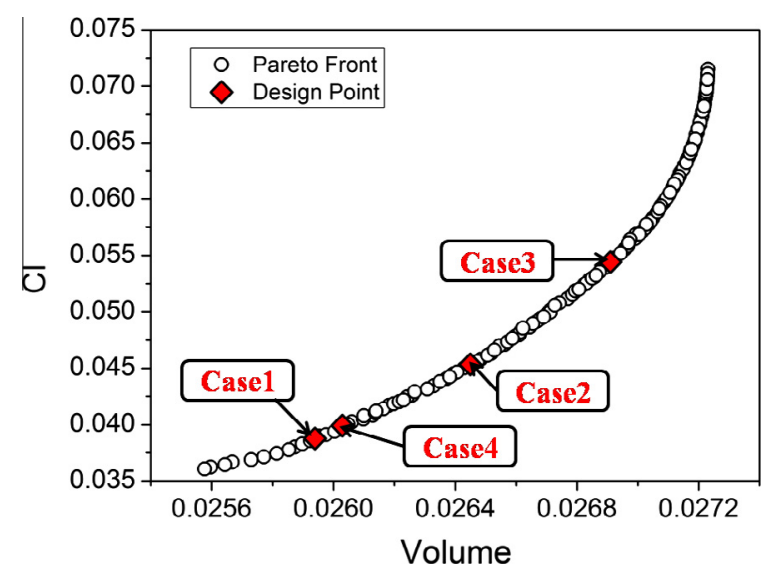

Fig. 10. The Pareto solution that is found based on Kriging model.

Table 4

The predication values and CFD values of typical design points.

\begin{tabular}{lllllll}
\hline & Real Vol & Kriging & Error $(\%)$ & Real $C_{l}$ & Kriging & Error (\%) \\
\hline Case1 & 0.02590 & 0.02594 & 0.154 & 0.04026 & 0.03877 & 3.701 \\
Case2 & 0.02644 & 0.02645 & 0.004 & 0.04624 & 0.04534 & 1.946 \\
Case3 & 0.02696 & 0.02691 & 0.185 & 0.05443 & 0.05441 & 0.004 \\
Case4 & 0.02598 & 0.02603 & 0.192 & 0.04066 & 0.03992 & 1.820 \\
\hline
\end{tabular}

\section{Results and discussion}

\subsection{Steady aerodynamics discussion}

The volume of the streamlined part ( Vol) and the lift force of the trailing car $\left(C_{l}\right)$ are treated as the optimization objectives, and the real-coded genetic approach with a population number of 300 and 1500 generations has been performed in the optimization process. Fig. 10 shows the Pareto front of the two objectives. It can be found that the volume of the optimal solutions is limited in a small zone, while $C_{l}$ varies a little bigger, indicating that $C_{l}$ is more sensitive to the change of aerodynamic shape. Four square spots are chosen in Fig. 10 as typical design points to validate the prediction accuracy of the Kriging model.

Table 4 shows the numerical results of the four typical design points and the prediction results of the Kriging model. It can be seen that the volume of Case 1 is slightly smaller than that of the original model, while the lift force of the trailing car is obviously smaller than that of the original model; the volume of Case 2 and
Table 5

The design parameters increment of typical design points (for 1:1 proportion).

\begin{tabular}{llllll}
\hline & W1 $(\mathrm{mm})$ & W2 $(\mathrm{mm})$ & W3 $(\mathrm{mm})$ & W4 $(\mathrm{mm})$ & W5 $(\mathrm{mm})$ \\
\hline Case1 & -85.5988 & 146.9707 & -84.9102 & 51.4608 & -1196.0931 \\
Case2 & -79.4602 & 170.1183 & 175.1482 & 54.8363 & -1189.1687 \\
Case3 & -32.6650 & 170.4103 & 405.4629 & 58.2826 & -1188.0350 \\
Case4 & -80.0637 & 168.8571 & -43.3648 & 43.3441 & -1189.5958 \\
\hline
\end{tabular}

Case 3 are both larger than that of the original model, while the lift force of Case 2 is a little smaller than that of the original model and the lift force of Case 3 is almost the same with that of the original model; for Case4, the volume changes little compared to the original model while the lift force decrease drastically. The volume results from the Kriging model keeps consistent with the computational results, with a maximum error of $0.192 \%$; the lift force from Kriging model is a little different from the numerical results, with a maximum error of $3.701 \%$, which still meets the engineering requirement. Consequently, it can be concluded that the Kriging model constructed in this paper could precisely reflect the relationship between the design variables and the objectives.

Fig. 11 shows the longitudinal profile and transverse profile of the four typical design points. Compared to the size of the whole train, the shape variations of the typical points are very little. The height of the nose is all lifted for four optimal models, while the inclination of the cowcatcher changes from forward to backward. Table 5 shows the variation of the design variables of the optimal models compared to those of the original model. It can be seen that the control point around the cowcatcher has been put backward more than $1 \mathrm{~m}$. Considering that $\mathrm{W} 3$ controls the width of the train body which directly influences on the volume of the train and W5 controls the drainage of the cowcatcher which has the least influence on the volume of the train, Case 4 has been chosen as the optimal shape and the comparison of the aerodynamic performance with the original model has been performed.

Table 6 shows the aerodynamic loads and the volume of the streamlined part for the original model and the typical optimal models. It can be seen that the aerodynamic performance of the four optimal models has been improved significantly, of which Case 1 obtains the best performance. The lift force of trailing car for Case 1 has been reduced by $26.19 \%$ and the drag of the whole train has dropped by $6.04 \%$, while the volume of the streamlined part has only been reduced by $0.23 \%$; The optimization objectives for Case2 are all improved compared to the original model. The volume of the streamlined part for Case 2 has been increased by $1.85 \%$, while the lift force of the trailing car and the aerodynamic drag of the whole train have been reduced by $15.38 \%$ and $3.86 \%$
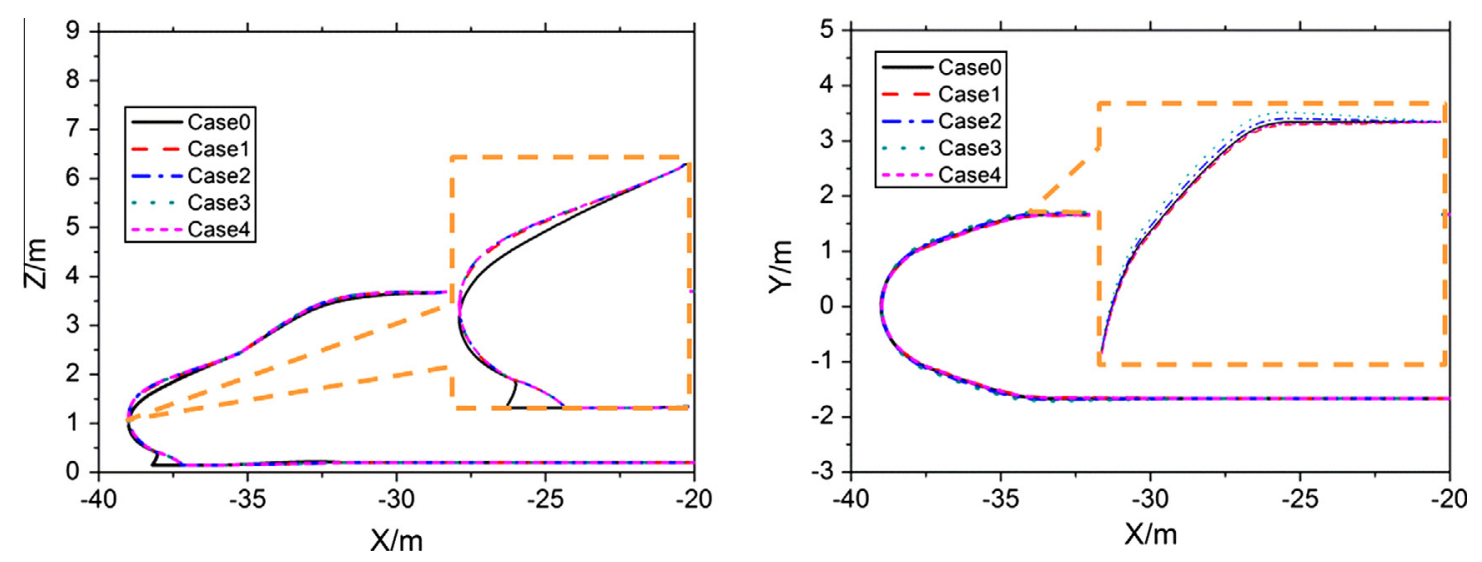

Fig. 11. The longitudinal and cross section line of typical design points. 
Table 6

The aerodynamic loads and the volume of the streamlined part for the original model and the typical optimal models.

\begin{tabular}{|c|c|c|c|c|c|}
\hline & $\operatorname{Vol}\left(\mathrm{m}^{3}\right)$ & $C_{l}$ & $C_{d}$ & Pressure & Shear \\
\hline Original & 89.7178 & 0.0546 & 0.1971 & 0.0659 & 0.1312 \\
\hline $\begin{array}{l}\text { Case1 } \\
\text { Reduction (\%) }\end{array}$ & $\begin{array}{l}89.5104 \\
0.23\end{array}$ & $\begin{array}{l}0.0403 \\
26.19\end{array}$ & $\begin{array}{l}0.1852 \\
6.04\end{array}$ & $\begin{array}{l}0.0530 \\
19.58\end{array}$ & $\begin{array}{l}0.1322 \\
0.76\end{array}$ \\
\hline $\begin{array}{l}\text { Case2 } \\
\text { Reduction (\%) }\end{array}$ & $\begin{array}{l}91.3766 \\
-1.85\end{array}$ & $\begin{array}{l}0.0462 \\
15.38\end{array}$ & $\begin{array}{l}0.1895 \\
3.86\end{array}$ & $\begin{array}{l}0.0563 \\
14.57\end{array}$ & $\begin{array}{l}0.1332 \\
1.52\end{array}$ \\
\hline $\begin{array}{l}\text { Case3 } \\
\text { Reduction (\%) }\end{array}$ & $\begin{array}{l}93.1738 \\
-3.71\end{array}$ & $\begin{array}{l}0.0544 \\
0.37\end{array}$ & $\begin{array}{l}0.1941 \\
1.52\end{array}$ & $\begin{array}{l}0.0612 \\
7.13\end{array}$ & $\begin{array}{l}0.1329 \\
1.30\end{array}$ \\
\hline $\begin{array}{l}\text { Case4 } \\
\text { Reduction (\%) }\end{array}$ & $\begin{array}{l}89.7869 \\
0.00\end{array}$ & $\begin{array}{l}0.0407 \\
25.46\end{array}$ & $\begin{array}{l}0.1858 \\
5.73\end{array}$ & $\begin{array}{l}0.0535 \\
18.82\end{array}$ & $\begin{array}{l}0.1323 \\
-0.84\end{array}$ \\
\hline
\end{tabular}

respectively. The aerodynamic lift force of Case3 keeps almost the same with the original model. However, the volume of the streamlined part for Case3 has been increased by $3.71 \%$ and the aerodynamic drag has been reduced by $1.52 \%$. For Case 4 , the volume of the streamlined part keeps unchanged with the original model, while the aerodynamic lift force and drag have been reduced by $25.46 \%$ and $5.73 \%$ respectively. The viscous drag is nearly the same with the inviscid drag for the simplified model, which takes significant role in the whole drag. However, the inviscid drag for the four typical optimal models is all reduced while the viscous drag keeps unchanged, indicating that the inviscid drag should be mostly focused for the optimization of aerodynamic drag.

Fig. 12 shows the pressure coefficient $\left(C_{p}\right)$ on the longitudinal profile of the leading and trailing streamlined parts for the original model and four typical optimal models. It can be seen that small negative pressure zone exists for the models after optimization. Because of the backward shift of the cowcatcher, the pressure coefficient of the lower surface drops obviously. Due to the slow transition between the cowcatcher and the bottom, the flow around the cowcatcher does not accelerate as much as the flow around the cowcatcher before optimization. As a result, the peak of the negative pressure zone of the optimal models is lower than that of the original model. Notable pressure variation could be found on the trailing streamlined part. Pressure peaks exist on the edges of the cab window and a pressure valley exists on the middle of the cab window. Minimum pressure could be detected around the nose zone of the trailing streamlined part. Different aerodynamic shape experiences similar pressure peaks at the same place. However, a distinct difference of minimum pressure could be observed especially for Case3 which owns the lowest minimum pressure. Due to the existence of the bogie below the trailing car, severe pressure variation exists around this region. The pressure fluctuates fiercely around the cowcatcher before optimization and much more fiercely after optimization.

Fig. 13 shows the pressure contour around the streamlined part of Case 4 and the original shape. It can be observed that an apparent high pressure zone exists around the nose and cowcatcher, and a slightly lower high pressure zone exists around the connection zone between the nose and the window. Meanwhile, an apparent low pressure zone exists beneath the cowcatcher. After optimization, the cowcatcher is moved backward, which slows the accelerating process of the passing flow. As a result, the high pressure zone here is reduced. Meanwhile, as the height of the nose changes with the slope of the window, the high pressure zone around the connection area also is alleviated. Therefore, the inviscid force of the leading car reduces a lot.

Fig. 14 shows the velocity profile around the nose of the leading streamlined part for Case 4 and the original model. It can be seen that the velocity profile agrees well at $x=-38 \mathrm{~m}$ while difference exists for the other positions, which is mainly due to the position that locates around the drainage zone. After optimization, the aero-
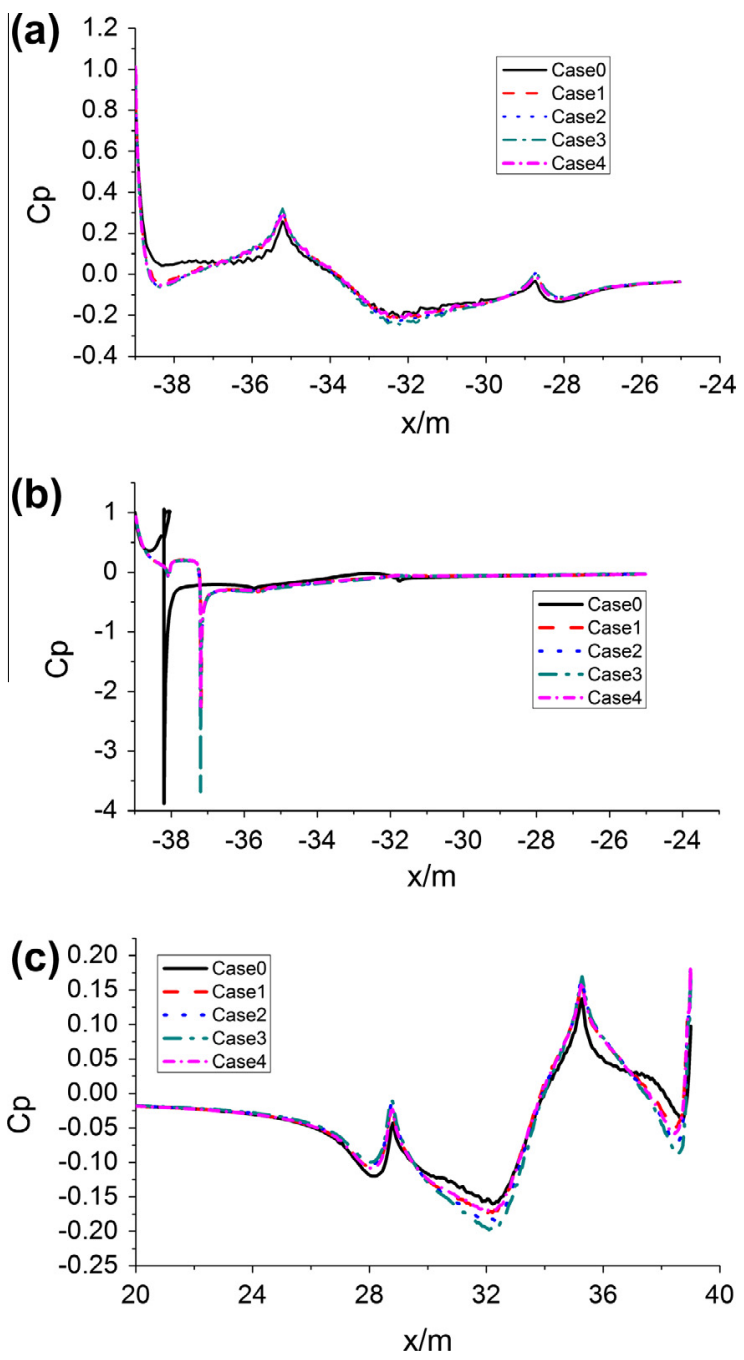

Fig. 12. The $C_{p}$ on the longitudinal profile of the original model and four typical optimal models.

dynamic shape has been slightly changed, resulting in the variation of flow status. Since little influence exists due to the deformation of the drainage zone at $x=-35 \mathrm{~m}$, the velocity profiles here for the original model and Case 4 are almost the same; at $x=-30 \mathrm{~m}$, the flow fluctuations due to the shape deformation get strengthened and the velocity profiles for the original model and Case 4 begin to vary away from each other; at $x=-27 \mathrm{~m}$, the flow has been smoothed by the transition zone, which weakens the influence due to shape deformation. As a result, the velocity profiles begin to be similar. 

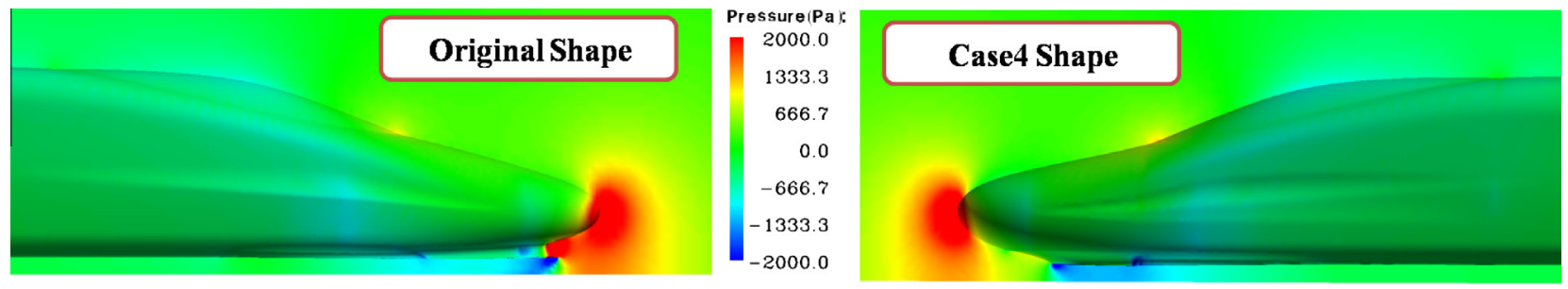

Fig. 13. Pressure distribution around the leading car before and after optimization.

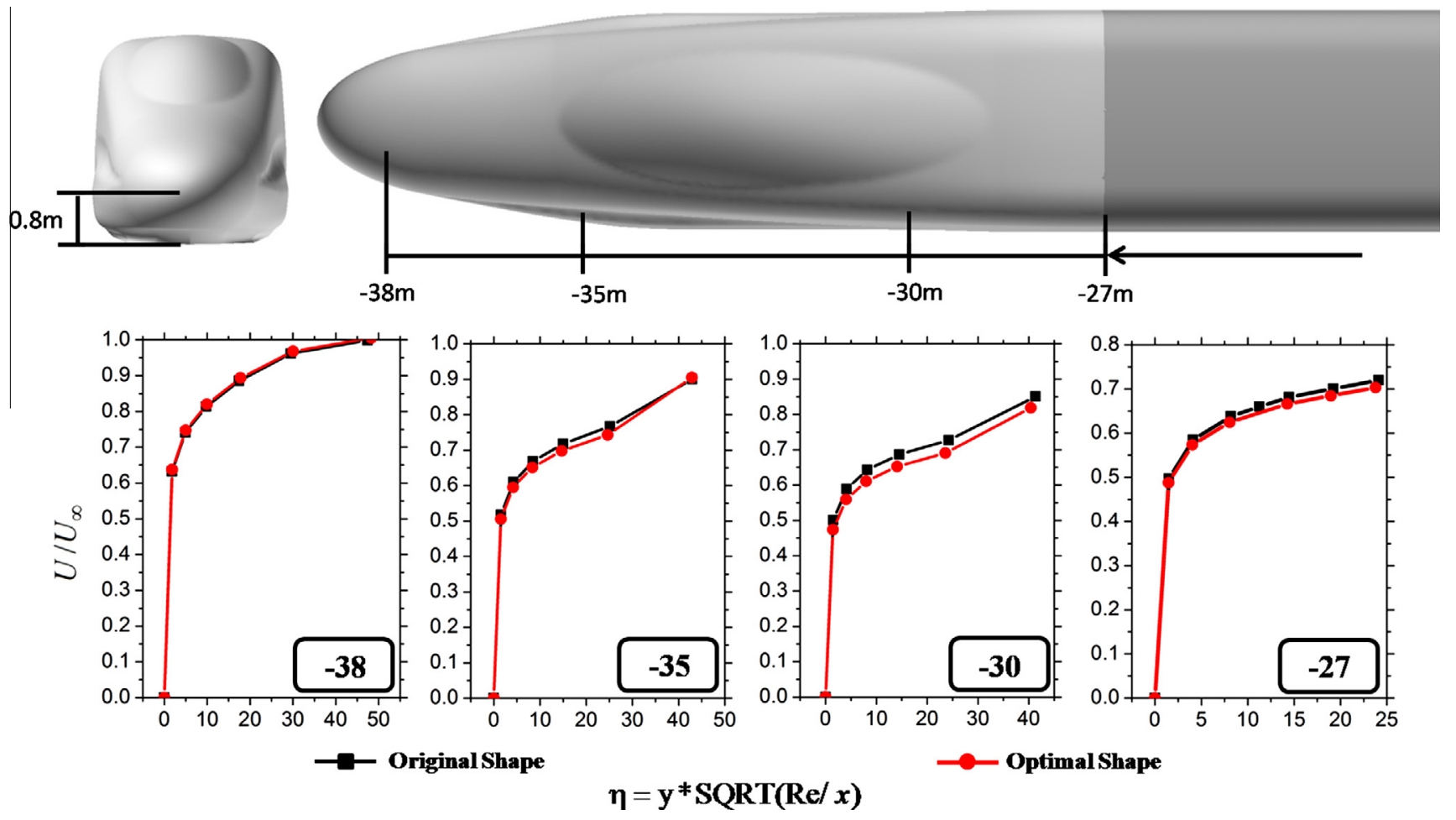

Fig. 14. The speed distribution around the nose before and after optimization.

\subsection{Aerodynamics discussion for real shape}

The above optimization of the streamlined shape is based on a simplified train, so that the cost of mesh generation and CFD analysis could be greatly reduced. A proper simplification on the CRH380A has been performed. Only the bogie beneath the streamlined part of the trailing car has been reserved while other ancillary components are all neglected. However, these components impose an intense effect on the aerodynamic performance of the whole train. In order to take the effect of the ancillary components into consideration and further study on the aerodynamic performance of the optimal train, a comparative discussion between the real shape of CRH380A and the optimal train (Case4) has been performed. The real models with all the ancillary components are shown in Fig. 15.

The CFD approach of the complicated models is the same as the simplified models. The hybrid mesh configuration has been adopted with the prism grids along the surface of the train and Cartesian grids in the outer of the prism grids. 6 prism layers have been generated with the initial height of $0.45 \mathrm{~mm}$ and a stretching ratio of 1.5. In order to obtain a smoother flow field, the zones around the ancillary components are locally densified. The total amount of the mesh is about 23 million.

The distribution of aerodynamic forces of the CRH380A prototype and the optimal train has been shown in Table 7. It can be obviously seen that the inviscid drag of the prototype CRH380A is greatly larger than the simplified one and the viscous drag is a little smaller. The inviscid drag of the first bogie beneath the leading streamlined part is slightly bigger, which is a result of the impact of the high-speed incoming flow. Due to the existence of a bogie cover under the streamlined part, the inviscid drag of the prototype CRH380A is greater than the simplified model. Large vortices are generated at the inter-spacing between adjacent cars which would produce negative drag on Shield- 1 and Shield- 3 on the leeward side and produce positive drag on Shiled-2 and Shield- 4 on the windward side. However, the algebraic sum of four shields is a small value, indicating that the inter-spacing imposes a great effect on the aerodynamic drag force distribution of each car while having little influence on the total aerodynamic drag force. The lift force of the trailing car arises from the pull force on the surface. However, due to negative lift force by the ancillary components, the total lift force of the trailing car of the prototype is a little smaller than the simplified one. The aerodynamic forces of the ancillary components keep basically unchanged before and after the optimization. With the same simplified model, the drag force of the leading and trailing noses of the complicated model gets reduced greatly, and also the lift force of the trailing streamlined part. Compared to the prototype CRH380A, the total drag force of the optimal train has been reduced by $2.95 \%$, in which the inviscid drag has been reduced by $5.13 \%$, and the viscous drag 


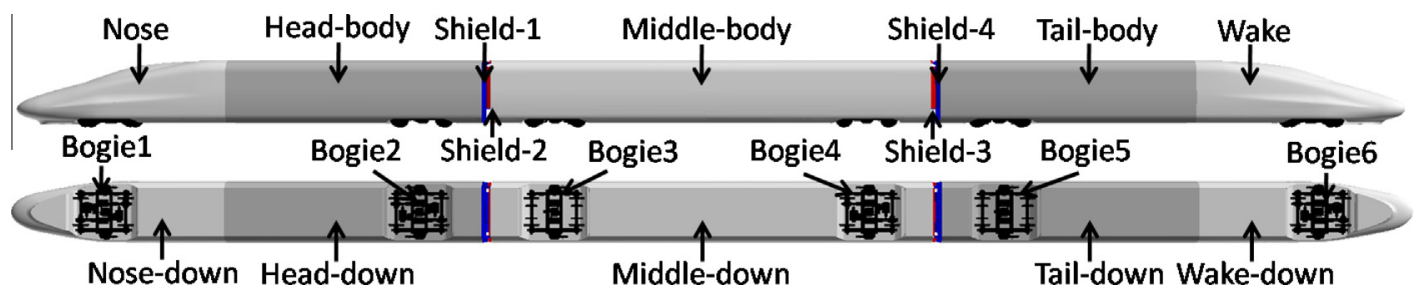

Fig. 15. The real shape of CRH380A.

Table 7

Drag and lift coefficient of different parts of CRH380A before and after optimization.

\begin{tabular}{|c|c|c|c|c|c|c|}
\hline \multirow[t]{2}{*}{ Train part } & \multicolumn{2}{|c|}{ Original shape } & \multicolumn{2}{|c|}{ Optimal shape } & \multirow{2}{*}{$\begin{array}{l}\text { Original shape } \\
\text { Tail } C_{l}\end{array}$} & \multirow{2}{*}{$\begin{array}{l}\text { Optimal shape } \\
\text { Tail } C_{l}\end{array}$} \\
\hline & Pressure & Shear & Pressure & Shear & & \\
\hline Nose & 0.0147 & 0.0145 & 0.0091 & 0.0152 & -ー-ー-ー-ー-ー & --ー-ー-ー- \\
\hline Nose-down & 0.0353 & 0.0023 & 0.0361 & 0.0020 & --ー-ー-ー--ー & -------- \\
\hline Head-body & 0.0000 & 0.0207 & 0.0000 & 0.0209 & -ーー-ーーーーーー & -ー-ーー-ー- \\
\hline Head-down & 0.0102 & 0.0026 & 0.0089 & 0.0024 & --ー--ー-ー-- & -------- \\
\hline Bogie1 & 0.0322 & 0.0011 & 0.0293 & 0.0012 & -ーーーーーーーーー & -ー-ー-ー-ー \\
\hline Bogie2 & 0.0075 & 0.0004 & 0.0076 & 0.0004 & --ー-ー-ー-ー- & ---ー---- \\
\hline Shield-1 & -0.0332 & 0.0001 & -0.0337 & 0.0000 & -ーーーーーーーーー & -ーー-ーーーー \\
\hline Middle-body & 0.0000 & 0.0320 & 0.0000 & 0.0325 & -ーー-ーー-ーーー & -ー-ーー-ーー \\
\hline Middle-down & 0.0113 & 0.0022 & 0.0134 & 0.0023 & -ー-ー-ー-ー-ー & --ー-ー-ー- \\
\hline Bogie3 & 0.0087 & 0.0004 & 0.0090 & 0.0004 & -ー-ー-ー-ーーー & --ー-ー-ー- \\
\hline Bogie4 & 0.0053 & 0.0002 & 0.0037 & 0.0002 & -ー-ー-ー-ー-ー & --ー-ー-ー- \\
\hline Shield-2 & 0.0485 & 0.0002 & 0.0489 & 0.0002 & -ーーーーーーーーー & -ー-ー-ー-ー- \\
\hline Shield-3 & -0.0279 & 0.0001 & -0.0309 & 0.0000 & --ー-ー-ー-ー- & -------- \\
\hline Tail-body & 0.0000 & 0.0180 & 0.0000 & 0.0183 & 0.0833 & 0.0843 \\
\hline Tail-down & 0.0042 & 0.0011 & 0.0050 & 0.0011 & -0.1194 & -0.1204 \\
\hline Wake & 0.0433 & 0.0107 & 0.0386 & 0.0111 & 0.1896 & 0.1767 \\
\hline Wake-down & 0.0081 & 0.0008 & 0.0038 & 0.0008 & -0.0899 & -0.0949 \\
\hline Bogie5 & 0.0042 & 0.0003 & 0.0047 & 0.0002 & -0.0061 & -0.0068 \\
\hline Bogie6 & 0.0043 & 0.0002 & 0.0041 & 0.0002 & -0.0062 & -0.0069 \\
\hline Shield-4 & 0.0423 & 0.0002 & 0.0459 & 0.0002 & -0.0147 & -0.0147 \\
\hline Total & 0.2143 & 0.1081 & 0.2033 & 0.1096 & 0.0366 & 0.0173 \\
\hline
\end{tabular}

increases by $1.39 \%$. The lift force of the trailing car has been reduced by $52.73 \%$, in which the trailing nose and the lower part of the streamlined part contribute the major part, reduced by $6.8 \%$ and 5.56\% respectively. Although the variation values of the simplified model and the prototype model are not the same, they both share the same tendency and could illustrate the effect on the aerodynamic force due to the change of the streamlined head. As a result, it is reasonable to utilize a simplified model to perform the optimization, just as the approach used in the present paper, which greatly reduces the cost of CFD analysis and improves the optimization efficiency.

\subsection{Unsteady aerodynamics discussion}

\subsubsection{Unsteady aerodynamics discussion without cross wind}

In order to compare the aerodynamic performance between the optimal model and the original model, the unsteady flow structures for both the models have been analyzed in the present paper in the condition with or without crosswind. This section mainly discusses the flow under conditions without crosswind and the next section mainly discusses the flow in crosswind condition. Table 8 shows the average value of the aerodynamic loads for the original model and the optimal model. It can be seen that the results from unsteady simulations are all a little smaller than those from steady simulation; after optimization, the aerodynamic drag force of the whole train has been reduced by $3.34 \%$ and the lift force of the trailing car has been reduced by $27.86 \%$, which shows the same tendency with the results from steady simulation. The aerodynamic performance of the optimal train has improved distinctly.
Table 8

The unsteady calculation results before and after optimization.

\begin{tabular}{lllc}
\hline & Case0 & Case4 & Reduction (\%) \\
\hline TOTAL-CD & 0.18969 & 0.18335 & 3.34 \\
TAIL-CL & 0.03535 & 0.02550 & 27.86 \\
\hline
\end{tabular}

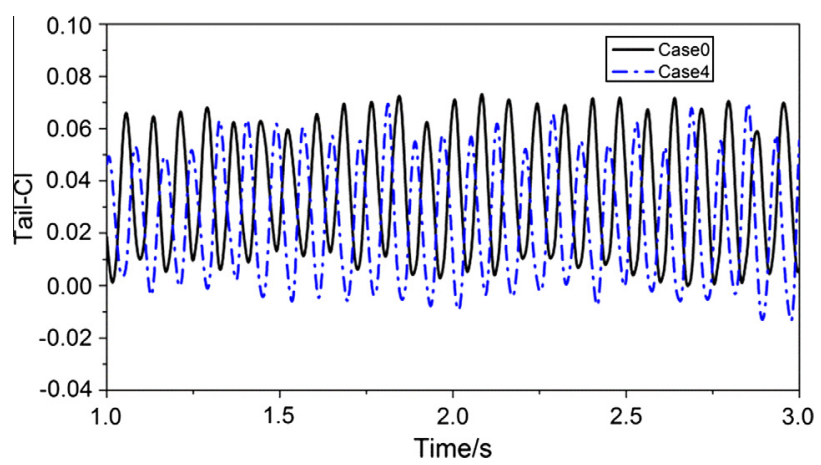

Fig. 16. Time history of the lift of the trailing car before and after optimization.

Fig. 16 shows the time history of the lift force of the trailing car for the trains before and after optimization. It can be seen that a significant difference exists. The maximum and minimum lift force of the original model (Case 0 ) is 0.0731 and -0.00008 respectively, with its amplitude of 0.07318 . For the optimal model (Case4), the maximum and minimum lift is 0.06995 and -0.01362 respectively, 

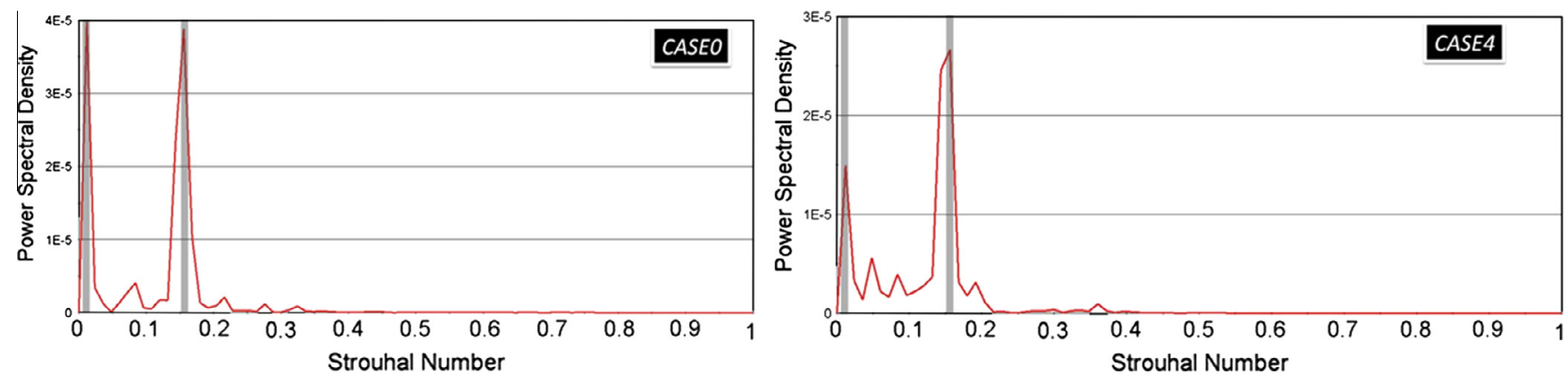

Fig. 17. Autopower spectra of the lift of the trailing car before and after optimization.
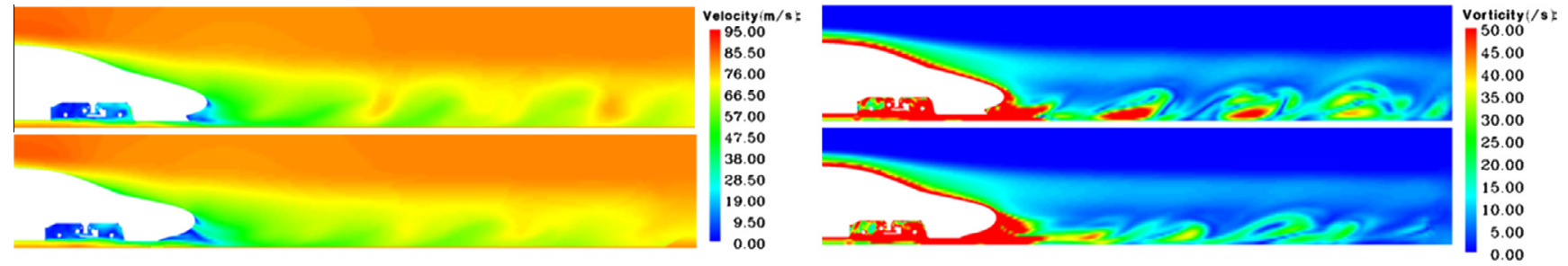

Fig. 18. Velocity and vorticity contour around the trailing car in $y=0$ section before and after optimization $(t=1.5 \mathrm{~s})$.

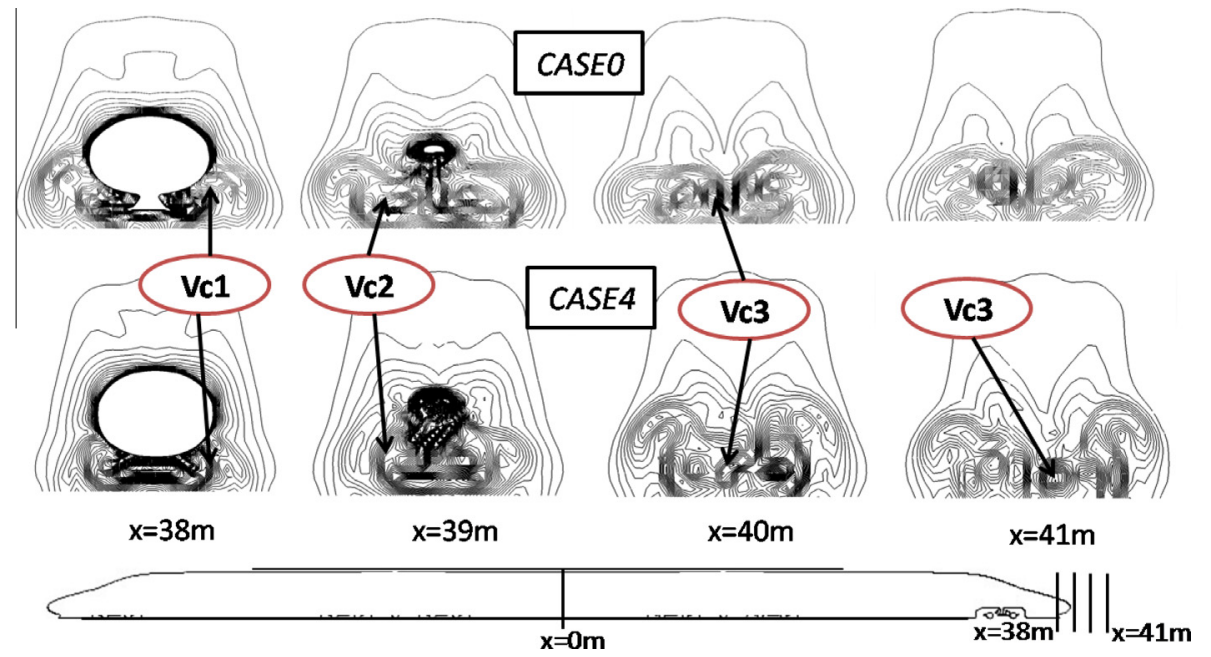

Fig. 19. Vorticity contour in different cross sections around the trailing car before and after optimization $(t=1.5 \mathrm{~s})$.

with its amplitude of 0.08357 , which is slightly larger than the original model. The maximum lift force of Case4 is obviously smaller than that of Case0, and the minimum lift force acts as a downward force, which is more favorable for the running safety and stability.

Fig. 17 shows the power spectral density of the lift force for the trains before and after optimization. It can be seen that one dominant frequency exists for the original train, which locates at $S t=0.012\left(S t=f D / U_{\infty}\right.$, where $D$ is the characteristic length, which is $1 \mathrm{~m}$, and $U_{\infty}$ is the inflow velocity), with a corresponding frequency of $1 \mathrm{~Hz}$. A secondary frequency could be observed at $S t=0.157$, with a corresponding frequency of $13.1 \mathrm{~Hz}$; for the train after optimization, the dominant frequency exists at $S t=0.156$, with its frequency of $13 \mathrm{~Hz}$, and also a secondary frequency could be found at $S t=0.011$, with its frequency of $0.92 \mathrm{~Hz}$. Consequently, the fluctuating frequency of the lift force of the trailing car gets a little larger for the train after optimization. However, the amplitude of the lift force gets smaller than the original train, indicating that the running amenity could still be preserved. Meanwhile, the running safety of the optimal train has been improved.
The aerodynamic shape of HST influences distinctly on the formation and evolution of trailing vortices. Different streamlined shapes may lead to different detached structures. Fig. 18 shows the longitudinal velocity contour and vorticity contour of the trains before and after optimization. It could be seen that the flow disturbed by the bogies interferes at the trailing nose with the flow from the upper surface of the streamlined part and big vortices with strong intensity begin to form. After optimization, the cowcatcher has been moved backward significantly. The low velocity zone around the nose increases, and correspondingly the high pressure zone and the intensity of the vortices grow larger and stronger. As a result, the train around this zone experiences stronger pull and the lift force of the trailing car gets smaller. As the vortices evolve backward, the intensity of the vortices for the optimal train grow weaker and the vortex cores get smaller. However, the intensity of the trailing vortices and magnitude of vortex cores for the original train are still stronger, which may throw a threat to the surroundings. 

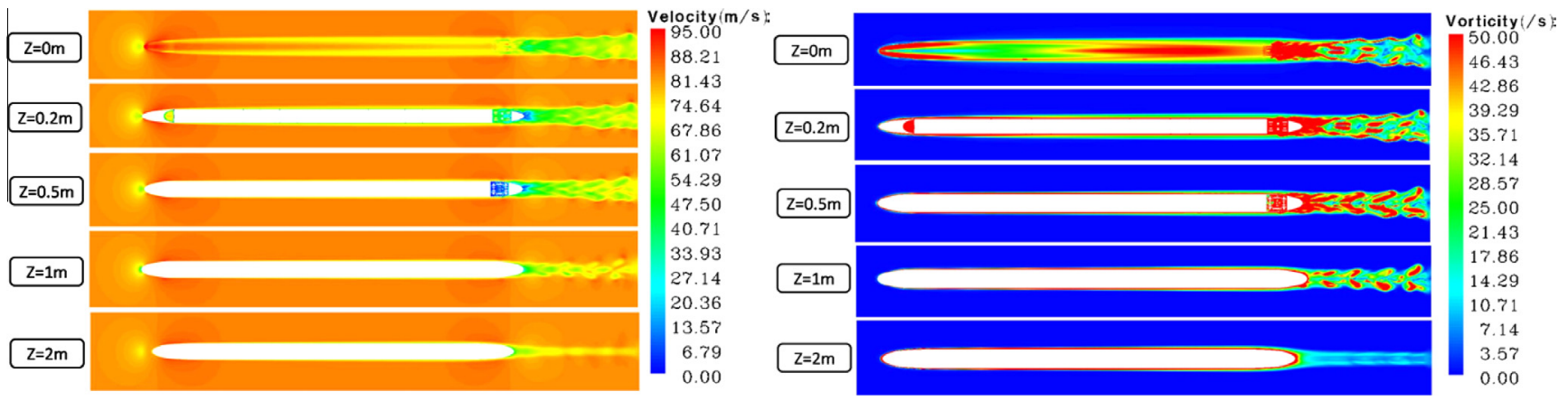

Fig. 20. Instantaneous velocity and vorticity contour in different sections in $z$ direction ( $t=1.5 \mathrm{~s}$, for original shape).
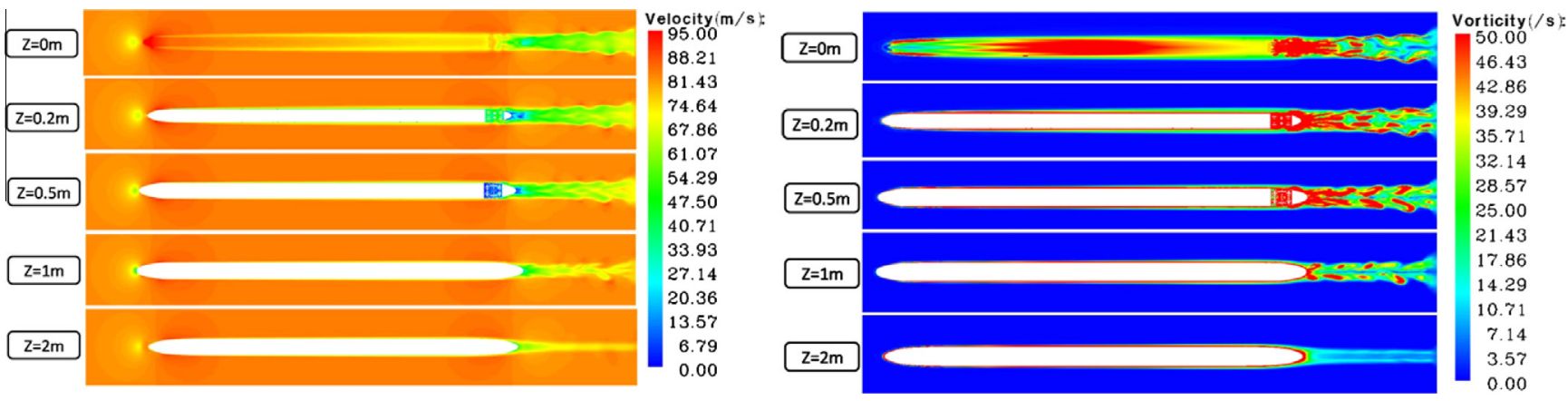

Fig. 21. Instantaneous velocity and vorticity contour in different sections in $z$ direction $(t=1.5 \mathrm{~s}$, for optimal shape).
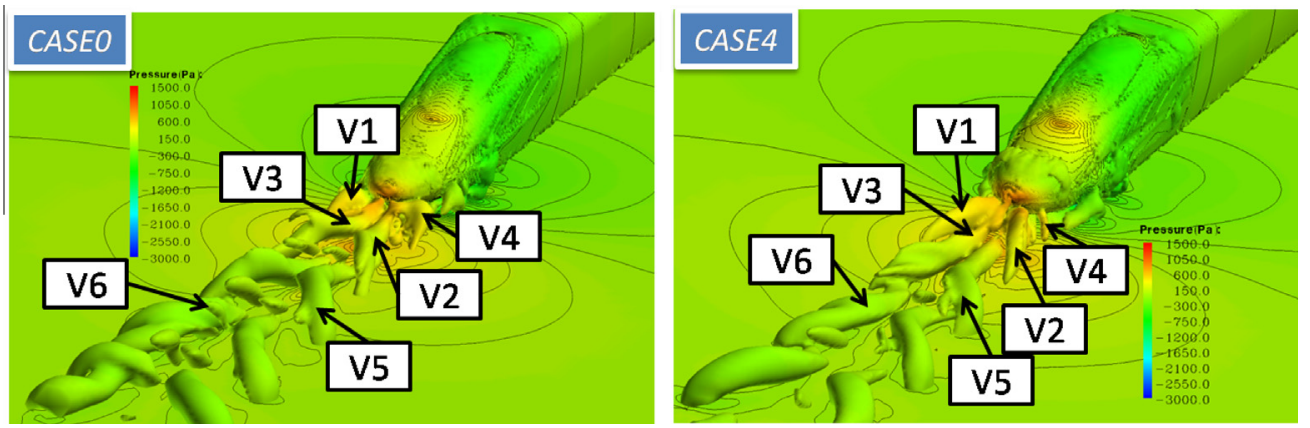

Fig. 22. Surface pressure contour of the trailing car and iso-surface of $Q$ before and after optimization $(t=1.5 \mathrm{~s}, Q=100)$.

In order to more clearly study the evolution of the trailing vortices for the trains before and after optimization, vorticity contour lines of different sections are shown in Fig. 19. It can be seen that two vortex structures Vc1 and Vc2 exist around the trailing nose, along the side of cowcatcher. A small vortex Vc3 begins to emerge at $x=40 \mathrm{~m}$, and still exists at $x=41 \mathrm{~m}$ for the optimal train while disappears for the original train. The scale of Vc1 and Vc2 gets smaller for the optimal train. They both converge to the middle at $x=39 \mathrm{~m}$ and spread to both sides in the wake zone.

Figs. 20 and 21 show the velocity contour and vorticity contour on different sections in the $z$ direction for the original train and optimal train. It can be seen that complicated flow structures exist around the ground. The formation and broken frequencies for the trailing vortices are very high. At $z=0.5 \mathrm{~m}$, the interaction between the trailing vortices and the ground gets weaker. However, the basic vortex structures are well preserved in this section. At $z=2 \mathrm{~m}$, the vortex structures get disappeared and the flow tends to stable; before optimization, the flow around the trailing nose seems more asymmetric, leading to the existence of side force. As the evolution of trailing vortices in the wake zone, the vortex pairs emerge symmetrically. For the optimal train, the asymmetric characteristics get weaker around the nose. The distance between the lateral vortex cores gets shorter, which could weaken the amplitude of the side force of the trailing car. As the development of the trailing vortices, the intensity of the trailing vortices gets weaker and weaker, and this may improve the running safety and stability of HST.

In order to directly study the space distribution of trailing vortices, the instantaneous iso-surface of $Q\left(Q=-1 / 2 \partial u_{i} / \partial x_{j} \partial u_{j} / \partial x_{i}\right)$ is shown in Fig. 22 for the trains before and after optimization. It could be seen that two big vortices develop from the sides of the trailing nose, and these vortices interact with the ground, resulting in smaller vortices. Four big vortices (V1, V2, V3 and V4) could be observed around the trailing nose. V4 gets very smaller for the optimal train. However, V4 gets disappeared after the interaction with the ground for the original train. V1 and V6 evolve together and form a new vortex V6, of which the intensity and vortex scale are stronger and bigger. The intensity of V2 is rather stronger for the original train and it interacts with the ground strongly. 
Table 9

The unsteady calculation results in cross wind condition before and after optimization.

\begin{tabular}{|c|c|c|c|c|c|c|}
\hline & TOTAL-CD & TAIL-CL & TAIL-CS & TAIL-MX & TAIL-MY & TAIL-MZ \\
\hline Case0 & 0.21773 & 0.26242 & -0.02153 & -0.02409 & -0.15518 & -1.21019 \\
\hline Case 4 & 0.21316 & 0.24817 & 0.00601 & -0.02816 & -0.16215 & -0.76195 \\
\hline Reduction (\%) & 2.10 & 5.43 & 72.09 & -16.89 & -4.49 & 37.04 \\
\hline
\end{tabular}

However, the intensity of V2 drops distinctly for the optimal train and disappears before it interacts with the ground. V5 evolve from the cowcatcher with strong intensity. It gets broken after interacting with the ground. V2 and V5 interact strongly before optimization. However, they hardly interact with each other for the train after optimization. The vortex core of V5 keeps well around the trailing nose, belonging to a kind of streamwise vortex structure.

\subsubsection{Unsteady aerodynamics discussion in cross wind condition}

Strong cross wind is frequently met in the real world, and the aerodynamic performance would deteriorate fiercely compared to the conditions without cross wind, which would impose a threat on the running safety. As a result, it is very necessary to study the aerodynamic performance of the trains before and after optimization in crosswind conditions. It is a key criterion to validate the optimal streamlined shape reasonable or not.

In the crosswind study, the running speed is set to be $300 \mathrm{~km} / \mathrm{h}$, and the crosswind velocity is $20 \mathrm{~m} / \mathrm{s}$ with an angle of $90^{\circ}$ compared to the running direction of the train. The CFD algorithm and mesh configuration are both the same with the calculation in condition without crosswind, except that the leeward side of the train has been densified so as to precisely capture the vortex structures there. The total amount of the grids is about 12 million.

Table 9 shows the average value of unsteady aerodynamic force for the trains before and after optimization in crosswind condition. It can be observed that the aerodynamic drag force of the whole train and the lift force of the trailing car have increased greatly compared to those in condition without crosswind, especially for the lift and side force of the trailing car, of which the magnitude has been increased by one order. After optimization, four of the six aerodynamic loads have been reduced, while the other two get increased in the absolute value. Since the optimization in the present paper is mainly focused on the aerodynamic lift and side force of the trailing car, the moment of the trailing train has not been considered during the optimization process. As a result, the increase of the overturning moment and yaw moment of the trailing car does not mean that the optimization results are unreasonable. Big reduction could be found for the side force and the pitching moment, which is reduced by $72.09 \%$ and $37.04 \%$ respectively. Meanwhile, the overturning moment has been increased by $16.89 \%$.

Fig. 23(a) shows the time history of the side force of the trailing car for the trains before and after optimization. Distinct difference could be observed between the trains. The side force keeps negative for the original train, with its minimum and maximum of -0.03844 and -0.00208 respectively. The amplitude of the side force of the original train is 0.03636 . However, the side force of the optimal train fluctuates around 0 , with its minimum and maximum of -0.000965 and 0.02924 respectively. Its amplitude is 0.03889 , which is larger than the original train. However, the absolute value of the side force of the optimal train has been reduced by $23.93 \%$, compared to the original train, which will greatly improve the running safety. Fig. 23(b) shows the time history of the lift force of the trailing car for the trains before and after optimization. The lift force for the optimal train tends to fluctuate nearer to 0 . The minimal and maximal lift force for the original train is 0.23921 and 0.29111 respectively, with its amplitude of 0.0519 .
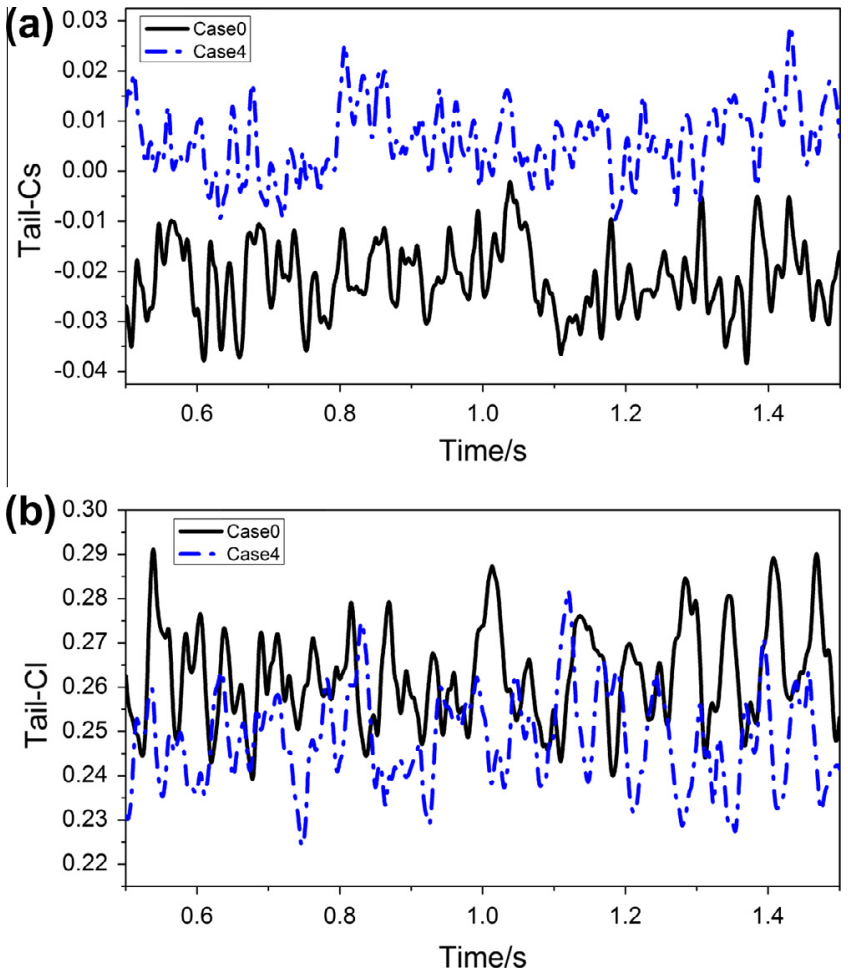

Fig. 23. Time history of the trailing car aerodynamics loads in cross wind condition before and after optimization.

After optimization these values change to 0.2241 and 0.28179 , with its amplitude of 0.05769 . The same conclusion could be drawn that optimal train will aid in the running safety but may slightly deteriorate the running amenity.

Fig. 24 shows the power spectral density of the side force of the trailing car for the trains before and after optimization. One dominant frequency could be observed for both the trains at $S t=0.011$, with a corresponding value of $0.92 \mathrm{~Hz}$. Compared to the results from conditions without crosswind, the value of secondary frequencies gets smaller. However more secondary frequencies could be observed. Four secondary frequencies exist at $S t=0.096$, $S t=0.192, S t=0.217, S t=0.275$ for the original train, with their corresponding frequencies of $8 \mathrm{~Hz}, 16 \mathrm{~Hz}, 18.08 \mathrm{~Hz}$ and $22.92 \mathrm{~Hz}$ respectively. Seven secondary frequencies exist at $S t=0.036$, $S t=0.060, S t=0.097, S t=0.161, S t=0.288, S t=0.467, S t=0.480$ for the optimal train, with their corresponding frequencies of $3 \mathrm{~Hz}, 5 \mathrm{~Hz}, 8.08 \mathrm{~Hz}, 13.42 \mathrm{~Hz}, 24 \mathrm{~Hz}, 38.92 \mathrm{~Hz}$ and $40 \mathrm{~Hz}$ respectively. From the view of frequency distribution, the fluctuation period of the train body tends to be longer in strong crosswind conditions. However, the magnitude of the higher frequencies within the longer period grows bigger, which deteriorates the running comfort. After optimization, due to the decrease of side force of the trailing car, the fluctuation of the train body of the trailing car tends to grow weak, which will be favorable to the running comfort.

Fig. 25 shows the power spectral density of the lift force of the trailing car for the trains before and after optimization. One 

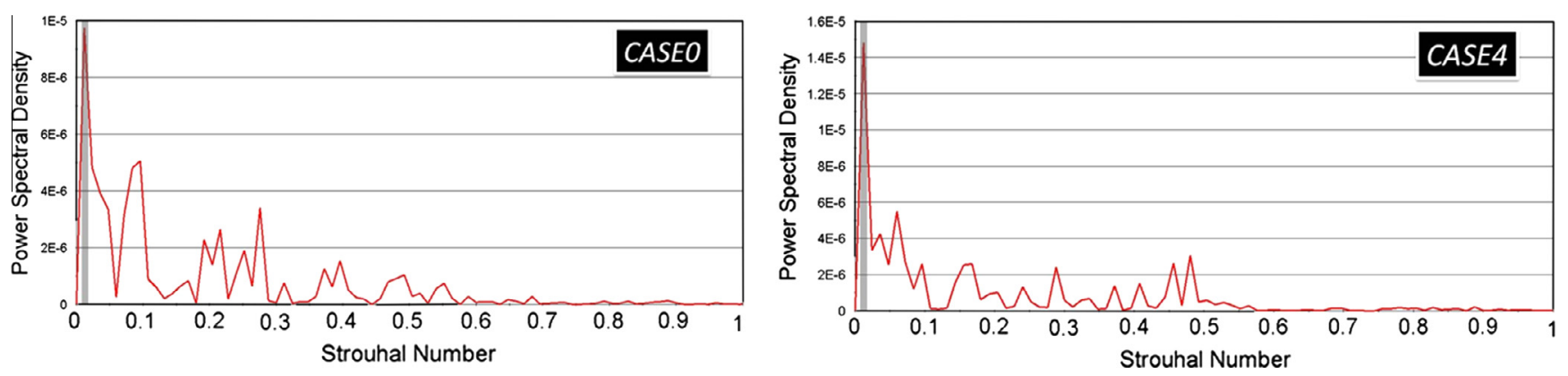

Fig. 24. Autopower spectra of the trailing car lift force in cross wind condition before and after optimization.
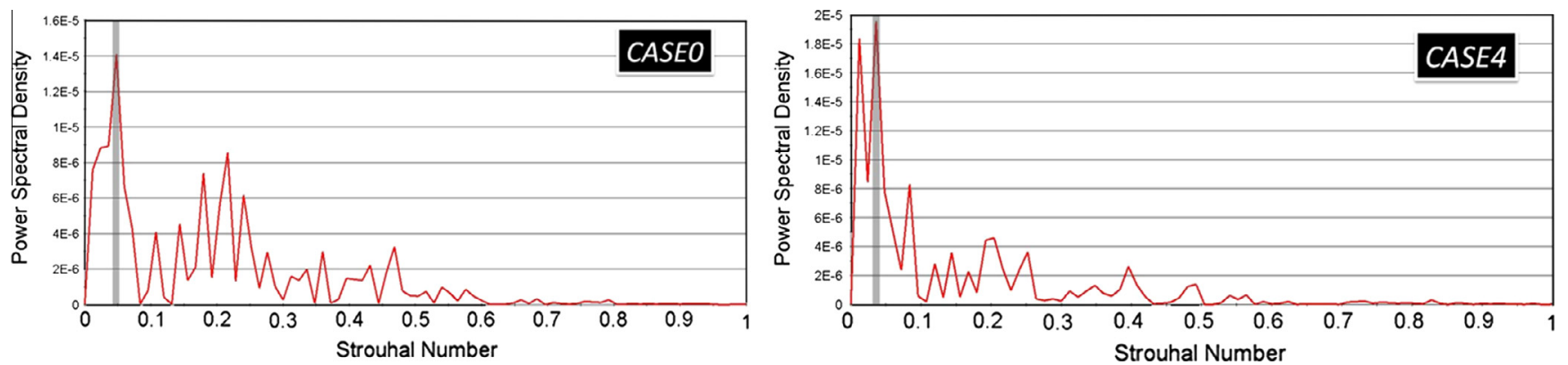

Fig. 25. Autopower spectra of the trailing car side force in cross wind condition before and after optimization.

dominant frequency could be observed for the prototype at $S t=0.048$, with a corresponding value of $4 \mathrm{~Hz}$. Meanwhile, a dominant frequency and corresponding value of $S t=0.036$ and $3 \mathrm{~Hz}$ could be found for the optimal train. The same with the results of the side force, in crosswind conditions, more secondary frequencies could be observed.

Eight secondary frequencies exist at $S t=0.109, S t=0.143$, $S t=0.18, S t=0.217, S t=0.240, S t=0.275, S t=0.360, S t=0.469$ for the original train, with their corresponding frequencies of $9.08 \mathrm{~Hz}, 11.92 \mathrm{~Hz}, 15 \mathrm{~Hz}, 18.08 \mathrm{~Hz}, 20 \mathrm{~Hz}, 22.92 \mathrm{~Hz}, 30 \mathrm{~Hz}$ and $39.08 \mathrm{~Hz}$ respectively. Eight secondary frequencies exist at $S t=0.012, S t=0.083, S t=0.120, S t=0.144, S t=0.168, S t=0.204$,
$S t=0.252, S t=0.397$ for the optimal train, with their corresponding frequencies of $1 \mathrm{~Hz}, 6.92 \mathrm{~Hz}, 10 \mathrm{~Hz}, 12 \mathrm{~Hz}, 14 \mathrm{~Hz}, 17 \mathrm{~Hz}, 21 \mathrm{~Hz}$ and $33.08 \mathrm{~Hz}$ respectively. From the view of frequency distribution, the fluctuation period of the train body tends to be longer in strong crosswind conditions. However, the magnitude of the higher frequencies within the longer period grows bigger, which deteriorates the running comfort. After optimization, due to the decrease of side force of the trailing car, the fluctuation of the train body of the trailing car tends to grow weak, which will be favorable to the running comfort.

Fig. 26 shows the instantaneous iso-surface of the second invariant of the velocity gradient $Q$ in the wake flow for both the

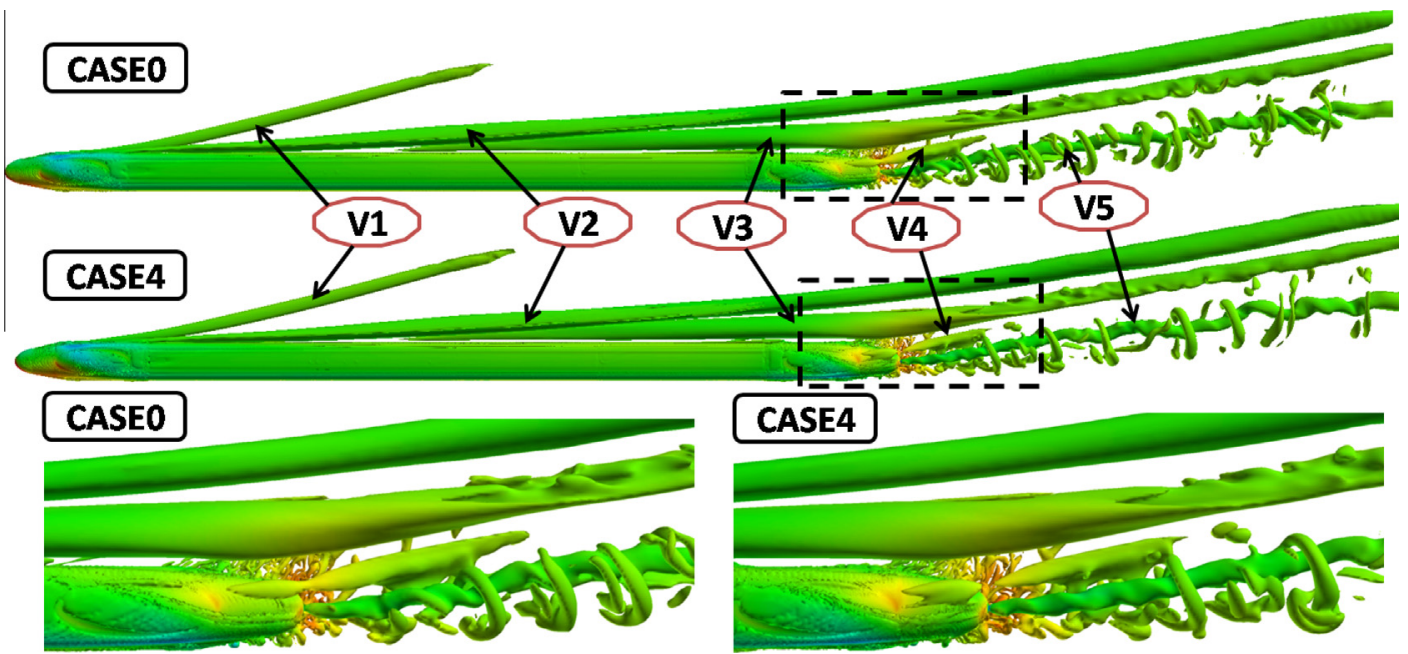

Fig. 26. Iso-surface of $Q$ around the high speed train in cross wind condition before and after optimization $(t=1.5 \mathrm{~s}, Q=100)$. 


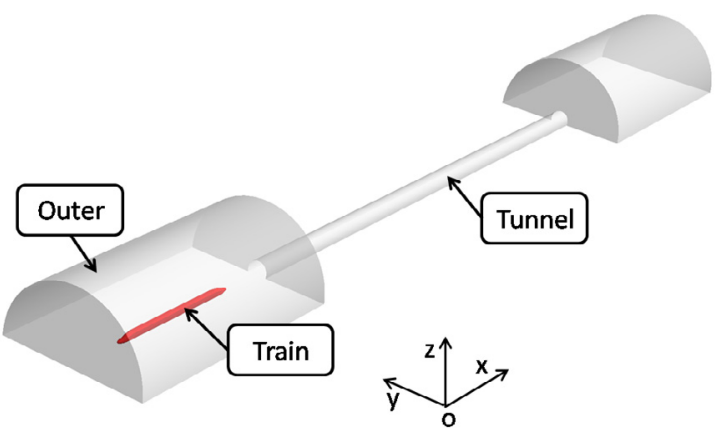

(a)

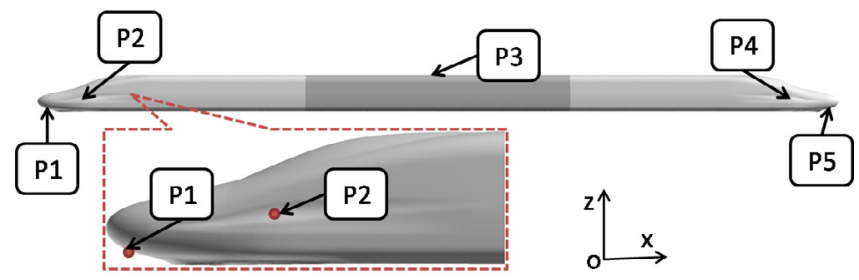

(b)

Fig. 27. (a) the schematic of the tunnel model and (b) the distribution of the pressure measuring points on the train body.

original train and the optimal train. It could be seen that three big vortex systems V1, V2 and V3 exist on the leeward side of the train, which all generate and evolve from the upstream streamlined head. V1 propagates in a helical mode and disappears in the middle part of the train. Meanwhile, V2 and V3 propagate along the train and still could be observed in the wake zone. As seen in the wake zone, the vortex structure of V3 is the biggest among these vortices, and gradually grows weaker as propagating downstream. V2 locates just outside V3, and propagates in parallel with V3 and V5. The vortices V4 and V5 develop from the nose of the trailing streamline, and V4 collapses rapidly due to the interaction of V3 and V5. V5 is a rather complicated vortex system among all the vortices, of which the vortex core is relatively small and a series of small horseshoe vortices exist along the vortex core of V5, which strengthens the influence range of V5. After optimization, the number of horseshoe vortices gets reduced and the intensity of V4 tends weaker in some extent. The vortex core of V5 gets narrower at the nose of the trailing car. The first horseshoe vortex along V5 moves backward compared to the flow structure of the original train, and a much more obvious distance of the second horseshoe vortex could be observed for the optimal train, which weakens the complexity of the wake flow.

\subsection{Aerodynamics for trains passing a tunnel}

The pressure wave will be formed when HST pass through a tunnel. Complicated pressure waves are generated due to propagation, reflection and superposition of the existing waves in the tunnel, which seriously influence the aerodynamic performance of HST. In addition, the compression waves that reach the tunnel exit at the sound velocity will suddenly swell so that a strong pulse wave known as micro-pressure wave is formed. The pulse wave may cause serious noise pollution and vibration damage to the surrounding environment. In this paper, the shape of leading car is the same as the trailing car, and the shape of leading car may have a serious effect on the pressure waves and micro-pressure wave.
Therefore, the aerodynamic performance for the original and optimal shape passing a tunnel will be discussed in this section.

The main factors that affect the intensity of the pressure wave in the tunnel are as follows: the blockage ratio, the buffer structure at the entrance and exit of the tunnel, the vertical shafts in the tunnel, the running speed of HST and the shape of the train head. In this paper, we just focus on the shape of the train head and do not study the influence of the tunnel structure as well as the ancillary components of the train in order to reduce the computational complexity. The speed of the train is $300 \mathrm{~km} / \mathrm{h}$. The cross-sectional area of the tunnel is $70 \mathrm{~m}^{2}$. The maximum cross-sectional area of the original shape is the same as the optimal shape, so the blockage ratio is a constant number as 0.16 . The worst tunnel length can be obtained through the following formula [21]:

$\frac{L_{t}}{L_{v}}=\frac{1}{M}$

where $L_{t}$ is the worst length of the tunnel, $L_{v}$ is the length of the train, $M$ is the Mach number. Thus, the worst tunnel length in this paper is $306 \mathrm{~m}$, which will be used as the tunnel length. Fig. 27(a) shows the schematic of the tunnel model, Fig. 27(b) shows the distribution of the pressure measuring points on the train body.

Fig. 28(a-e) shows the pressure histories of the measuring points. After optimization, the maximum pressure values of P1, P2 and P4 increase a little, while the absolute values of the minimum pressure values decrease a little. Thus, the pressure magnitude values of the three points are almost unchanged. Besides, the pressure histories of P3 and P5 are same before and after optimization. The micro-pressure waves of the original and optimal shape that are obtained $10 \mathrm{~m}$ away from the exit of the tunnel are shown as Fig. 28(f). As can be seen, the micro-pressure wave changes little before and after optimization. It means that small deformation of the train head has little effect on the pressure waves that are formed when a train passing a tunnel.

Pressure variations could affect the passengers' amenity as long as the variation is serious enough in a certain time interval. When the train passes by the tunnel, pressure waves generate not only in the tunnel but also inside the train. After obtaining the pressure history outside the train, the pressure variation inside the train could be obtained as long as the airtight coefficient of the train is known. Consequently, only the pressure variation outside the train will be discussed. in the paper As seen above, the corresponding place on both trains own similar pressure variations, not only the pressure pattern but also the pressure amplitude. As a result, the original shape is selected for the analysis of the pressure variation. As seen in Fig. 28, P1 and P2 belong to the leading car. During the passage of the train, the maximum pressure variation is up to $3445 \mathrm{~Pa}$. Since the whole time for the train passing is about $4.5 \mathrm{~s}$, the maximum pressure variation in $4 \mathrm{~s}$ or $10 \mathrm{~s}$ is the same to the former. The biggest pressure variation in 1s is about $3423 \mathrm{~Pa}$, and the maximum pressure gradient is about $3738 \mathrm{~Pa} / \mathrm{s}$. As for P3 in the middle of the train, the corresponding values are $3264 \mathrm{~Pa}$, $3150 \mathrm{~Pa}$ and $3792 \mathrm{~Pa} / \mathrm{s}$ respectively. As for P4 and P5 on the trailing car, the corresponding values are $3264 \mathrm{~Pa}, 3150 \mathrm{~Pa}$ and $3792 \mathrm{~Pa} / \mathrm{s}$ respectively. Since the airtight coefficient of the train is unknown, the pressure variation inside the train could not be analyzed. However, the above values could still be useful for the further assessment of passengers' amenity as long as the airtight coefficient of the train is obtained.

\section{Conclusions}

The complex wake flow of HST severely influences on the running safety and amenity of the trailing car. In order to improve the aerodynamic performance of the trailing car and weaken the 

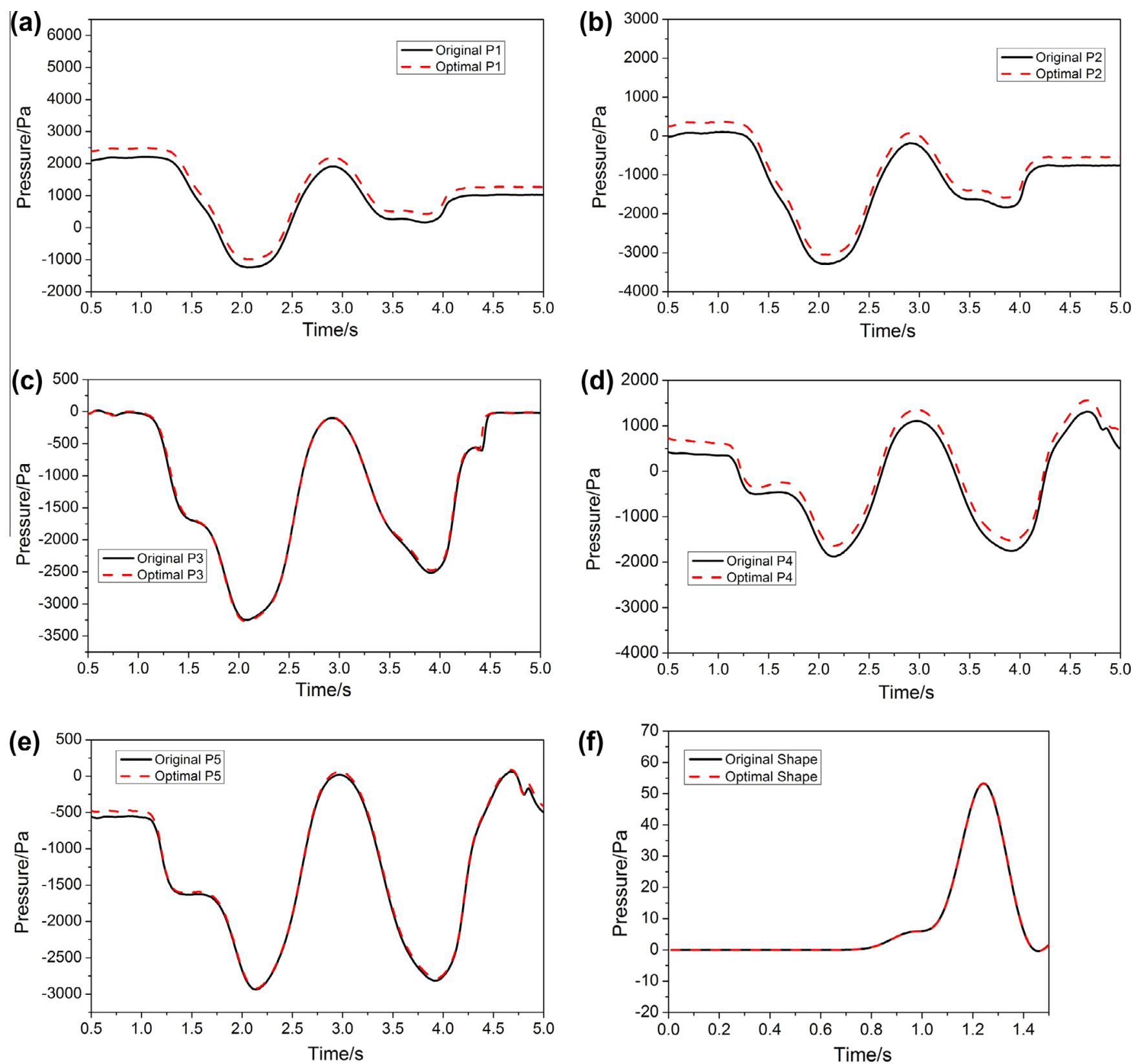

(f)

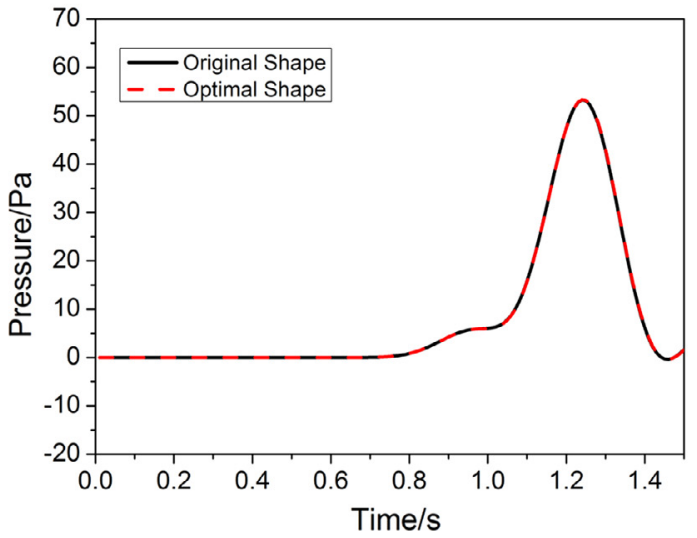

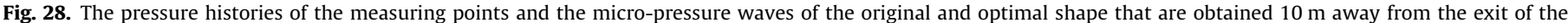
tunnel.

influence of the wake flow, a multi-objective optimization on the CRH380A streamlined head has been performed in the present paper, taking the lift force of the trailing car and the volume of the streamlined part as the optimization objectives, and a complete high efficiency optimization strategy based on the RSM has been constructed. This work could provide guidance to the aerodynamic shape optimization of high speed trains.

As a large aspect ratio transportation vehicle, a three-carriage high speed train has been utilized in the present paper in order to precisely obtain the aerodynamic loads of the trailing car. Since only the streamlined part is under investigation, only the bogie below the streamlined part of trailing car has been considered so as to reduce the computational time. After optimization, the aerodynamic performance of the train with all the bogies has been comparatively analyzed.

Due to the heavy computational cost ( $20 \mathrm{~h}$ should be ensured for a steady simulation of the flow field with 60 CPUs), an approach based on the combination of cross-validation method and the GA method has been proposed in the present paper to construct the Kriging model, which could fully utilize the information of sample points and greatly reduce the number of training samples. By the comparative analysis of test functions, it could be found that this approach could highly improve the optimization efficiency in the condition without reducing the generalization capability of the model. Five key design variables have been extracted for the streamlined part of HST and the Kriging model which satisfies the engineering requirement is constructed with only 23 sample points.

After obtaining the Pareto optimal set based on the lift force of the trailing car and the volume of the streamlined part, four typical optimal solutions have been chosen for a comparative study with the prototype, and one typical solution has been selected for an unsteady flow field analysis. Results reveal that the variation of the lift and side force of the trailing car, the drag force of the whole 
train in the design space tends to be the same. After optimization, the aerodynamic drag force of the whole train has been reduced by $3.34 \%$ and the lift force of the trailing car has been reduced by $27.86 \%$. In crosswind conditions, the lift force of the trailing car decreases by $5.43 \%$, the side force of the whole train decreases by $72.09 \%$ and the drag force of the whole train decreases by $2.1 \%$. The optimal train benefits from low fluctuations of lift and side force. Besides, better wake flow could be obtained, and the wake vortices are suppressed. Consequently, high running safety and amenity could be attained for HST after optimization, indicating that the optimization strategy proposed in the present paper could be applied in the aerodynamic shape design of HST. In addition, optimization based on the steady flow field calculation could be favorable for the optimization of unsteady aerodynamic loads. The optimization results could be validated by the unsteady flow field analysis.

Many design objectives that have a serious effect on the running safety and amenity of HST should be considered during the optimization process. However, we only take the aerodynamic lift force of the trailing car and the volume of the streamlined part as the optimal objectives and discuss some other design objectives after the optimal shape is found. Thus, the optimal shape of the two optimal objectives may not be the best shape of other design objectives. Therefore, the multi-point and multi-objective optimization study should be done in the future in order to find a more reasonable shape for much more optimal objectives. Besides, when we simulate the flow flied around the HST with CFD tools, the wheel rotation movement is not taken into consideration, which may influence the optimal results, so more reasonable CFD methods should be used for aerodynamics shape optimization of HST. What's more, although we show that it is suitable to use the simplified shape for HST optimization, the real shape is more reasonable during the optimization process so as to get better train nose shapes based on different optimal objectives.

\section{Acknowledgements}

This work was supported by 973 program (2011CB711100) and national key technology R\&D program (2009BAQG12A03). Computing Facility for Computational Mechanics Institute of Mechanics, Chinese Academy of Sciences is gratefully acknowledged.

\section{References}

[1] Baker C. The flow around high speed trains. J Wind Eng Ind Aerodynam 2010;98(6-7):277-98.

[2] Kim I, Ok H. A study on the aerodynamic characteristics of a high speed train entering a tunnel and frontal shape optimization. J KSAS 1998;26(1):17-26.

[3] Lee Jongsoo, Kim Junghui. Approximate optimization of high-speed train nose shape for reducing micropressure wave. Ind Appl 2008;35:79-87.

[4] Vytla VV, Huang PG, Penmetsa RC. Multi objective aerodynamic shape optimization of high speed train nose using adaptive surrogate model. AIAA2010-4383.

[5] Ku Y-C, Kwak M-H, Park H-I, Lee D-H, Multi-objective optimization of highspeed train nose shape using the vehicle modeling function. In: 48th AIAA aerospace sciences meeting, Orlando, USA; 2010.

[6] Krajnovic S. Shape optimization of high-speed trains for improved aerodynamic performance. P I Mech Eng F - J Rai 2009;223:439-52.

[7] Cui K, Wang XP, Hu SC, et al. Shape optimization of high-speed train with the speed of $500 \mathrm{kph}$. In: Lecture notes in electrical engineering - proceedings of the 1 st international workshop on high-speed and intercity railways, vol. 2 . Tiergartenstrasse 17, Heidelberg, D-69121, Germany: Springer Verlag; 2012 [Lnee 148. p. 187-97].

[8] Ku YC, Rho JH, Yun SH, et al. Optimal cross-sectional area distribution of a high-speed train nose to minimize the tunnel micro-pressure wave. Struct Multidiscip Optimiz 2010;42:965-76.

[9] Yao Shuanbao, Guo Dilong, Yang Guowei. Three-dimensional optimization design of high-speed train nose based on GA-GRNN. Sci China Technol Sci 2012;55(11):3118-30.

[10] Yao Shuanbao, Guo Dilong, Sun Zhenxu, et al. Multi-objective optimization of the streamlined head of high-speed trains based on the Kriging model. Sci China Technol Sci 2012;55(12):3494-508.

[11] Sun ZhenXu, Song JingJing, An YiRan. Optimization of the head shape of the CRH3 high speed train. Sci China 2010;12:3356-64.

[12] Blazek J. Computational fluid dynamics principles and applications. Elsevier Ltd.; 2005. p. 16-7.

[13] Yan C. Method and application of computational fluid dynamics. Beijing: Beihang University Press; 2006. p. 236-7.

[14] Nishino T, Roberts GT, Zhang X. Unsteady RANS and detached-eddy simulations of flow around a circular cylinder in ground effect. J Fluids Struct 2008;24:18-33.

[15] Spalart Philippe R. Detached-eddy simulation. Ann Rev Fluid Mech 2009;41:181-202.

[16] Chiu TW. Prediction of the aerodynamic loads on a railway train in a crosswind at large yaw angles using an integrated two and three-dimensional source/vortex panel method. J Wind Eng Ind Aerodynam 1995;57:47-74.

[17] Hemida Hassan, Krajnovic Sinisa. LES study of the influence of the nose shape and yaw angles on flow structures around trains. J Wind Eng Ind Aerodynam 2010;98:34-46.

[18] Jones Donald R. A taxonomy of global optimization methods based on response surfaces. J Glob Optimiz 2001;21:345-83.

[19] Coello CA, Lechuga MS. MOPSO: a proposal for multiple objective particle swarm optimization. In: Proceedings of the congress on evolutionary computation (CEC'2002), Honolulu, Hawaii, USA; 2002. p. 1051-6.

[20] Grosso A. Finding maximin Latin hypercube designs by iterated local search heuristics. Eur J Oper Res 2009;197(2):541-7.

[21] Wang YW, Yang GW, Huang CG. Influence of tunnel length on the pressure wave generated by high-speed trains passing each other. Sci China Technol Sci 2012;55:255-63. 